\title{
Preclinical Evidence and Possible Mechanisms of Extracts or Compounds from Cistanches for Alzheimer's Disease
}

\author{
Xiao-Li Zhou, Meng-Bei Xu, Ting-Yu Jin, Pei-Qing Rong, Guo-Qing Zheng*, Yan Lin* \\ Department of Neurology, the Second Affiliated Hospital and Yuying Children's Hospital of Wenzhou Medical \\ University, Wenzhou, China
}

[Received April 23, 2018; Revised August 12, 2018; Accepted August 15, 2018]

\begin{abstract}
Currently, disease-modified strategies to prevent, halt or reverse the progress of Alzheimer's disease (AD) are still lacking. Previous studies indicated extracts or compounds from Cistanches (ECC) exert a potential neuroprotective effect against AD. Thus, we conducted a preclinical systematic review to assess preclinical evidence and possible mechanisms of ECC in experimental AD. A systematical searching strategy was carried out across seven databases from their inceptions to July 2018. Twenty studies with 1696 rats or mice were involved. Neurobehavioral function indices as primary outcome measures were established by the Morris water maze test $(n=11)$, step-down test $(n=10)$, electrical $Y$-maze test $(n=4)$, step-through test $(n=3)$, open field test $(n=2)$ and passage water maze test $(n=1)$. Compared with controls, the results of the meta-analysis showed ECC exerted a significant effect in decreasing the escape latency, error times and wrong reaction latency in both the training test and the retention test, and in increasing the exact time and the percentage of time in the platformquadrant and the number of platform crossings (all $P<0.01$ ). In conclusion, ECC exert potential neuroprotective effects in experimental AD, mainly through mechanisms involving antioxidant stress and antiapoptosic effects, inhibiting $A \beta$ deposition and tau protein hyperphosphorylation and promoting synapse protection. Thus, ECC could be a candidate for AD treatment and further clinical trials.
\end{abstract}

Key words: Cistanches, Alzheimer's disease, dementia

\begin{abstract}
Alzheimer's disease (AD) is one of the most common, stubborn neurodegenerative disorders and is characterized by progressive cognitive dysfunction and behavioral impairment [1-3], accounting for $60 \sim 80 \%$ of all dementia cases [4]. In 2017, 5.3 million Americans aged 65 and older lived with AD; by 2050, this number will rise as high as 16 million in the US and 135 million worldwide. More than 15 million Americans provided an estimated 18.2 billion hours of unpaid care for patients with dementia, valued at more than $\$ 230$ billion; by 2050 , these costs could rise as high as $\$ 1.1$ trillion $[5,6]$. However, the cause of AD remains poorly understood. It is widely accepted that AD is associated with extracellular deposits
\end{abstract}

of amyloid $\beta(\mathrm{A} \beta)$ peptide and intracellular tau aggregates $[7,8]$. As apoptosis of neurons develops and connections among cells are lost, learning and memory impairment emerges and disease progresses $[9,10]$. The prescription drugs approved by the FDA in the US for AD symptom control include: (1) cholinesterase inhibitors (ChEIs) such as donepezil, galantamine, rivastigmine and huperzine A, which maintain average acetylcholine levels by reducing the activity of acetylcholinesterase and (2) N-methyl-Daspartic acid (NMDA) receptor antagonist, memantine, which protects neurons against excessive glutamate by partially blocking NMDA receptors [11]. However, all of them are only temporarily symptom relievers and can

*Correspondence should be addressed to: Drs. Guo-Qing Zheng and Yan Lin, the Second Affiliated Hospital and Yuying Children's Hospital of Wenzhou Medical University, Wenzhou, China. Email: gq_zheng@ sohu.com and Yan Lin. Email: linyanm@vip.sina.com.

Copyright: () 2018 Zhou XL et al. This is an open-access article distributed under the terms of the Creative Commons Attribution License, which permits unrestricted use, distribution, and reproduction in any medium, provided the original author and source are credited. 
bring undesirable side effects, such as headache, dizziness, nausea, vomiting, insomnia, other somatic symptoms and drug interactions [11-13]. Diseasemodified strategies to prevent, halt or reverse AD progress are urgently needed. There are rising numbers of $\mathrm{AD}$ patients seeking various kinds of complementary and alternative medicines worldwide, among which Chinese herbal medicines (CHMs) have high potential [14].

Herba Cistanches, a desert living Cistanche, Roucongrong, the dried fleshy stem of Cistanche deserticola $Y . \quad C$. Ma, first recorded in Shennongbencaojing (Shennong's Classic of Materia Medica, written about 475 B.C.-220 A.D.), is known as the desert ginseng and is of high medicinal value $[15,16]$. Showing a high antioxidative and antiinflammatory activity, Cistanches possess broad medicinal functions in neuroprotection, immunomodulation, endocrine regulation, hepatoprotection and bone-formation promotion. Nowadays, Cistanches is widely used in CHM formulas for treating various kinds of disorders, including aging and dementia [17-20]. Extracts or compounds from Cistanches (ECC), containing or representing the major bioactive ingredients, include Cistanches deserticola polysaccharides (CDPS), glycosides of Cistanches (GCs), and phenylethanoid glycosides (PhGs) such as echinacoside (ECH), acteoside (AS) and tubuloside B [21, 22]. Some preliminary clinical trials [23-26] indicated that ECC monotherapy for $\mathrm{AD}$ symptom control encouragingly received positive feedbacks. However, the effects of ECC and possible mechanisms behind these effects on AD remain uncertain. Furthermore, the clinical study is limited owing to various restrictions due to morality and methodology [27]. The systematic evaluation of preclinical researches is an essential method to integrate preclinical evidence and can be of high value in improving the quality of preclinical researches and guiding potential clinical translation and application [28, 29]. Thus, in the present study, we aim to conduct a preclinical systematic review of the efficacy of ECC and the mechanisms involved in experimental AD.

\section{MATERIALS AND METHODS}

\section{Search strategies}

Seven English and Chinese databases, including PubMed, the Cochrane Library, EMBASE, China National Knowledge Infrastructure (CNKI), VIP Journals Database, China Biology Medicine Database (CBM) and Wanfang Database, were electronically searched from their inceptions to July 2018. The following keywords were used: "Cistanche* OR Roucongrong" and "Alzheimer's disease OR dementia OR mild cognitive impairment". All studies were limited to animals.

\section{Eligibility criteria}

\section{Types of studies}

Animal studies that assess the effectiveness of ECC for $\mathrm{AD}$ were included, regardless of blinding, publication status or language. Reviews, comments, cases, clinical experiences or trials were excluded.

\section{Types of experimental animals}

Animal models of AD were included, regardless of animal species, gender, age and methods of model establishment. Models of other kinds of dementia, such as vascular dementia or Parkinson's disease, were excluded.

\section{Types of intervention and comparator}

Intervention versus comparator was as follows: A, ECC versus non-functional liquid/normal saline/no treatment; B, ECC versus western conventional medicine (WCM); C, ECC plus WCM versus WCM. ECC included CDPS, GCs and PhGs such as ECH, AS and tubuloside B, regardless of dose, form, administration method, or duration. However, ECC plus acupuncture/other CHMs versus acupuncture/other CHMs were excluded.

\section{Types of outcome measures}

The primary outcome measures were neurobehavioral function indices (NFIs) such as the Morris water maze test and step-down test. The secondary outcome measures were neurobiochemical and neuropathologic changes.

\section{Data extraction}

Two independent authors extracted data from the qualified articles according to a standardized data extraction form. The data of the highest dose were included when the treatment groups included various doses of the drug. The result of the peak time point was included when the data were expressed at different times. If published outcome data were demonstrated graphically, we made an effort to contact the author for further information. Digital ruler software was applied when a response was not received.

\section{Risk of bias in individual studies}

The risk of bias was assessed by the nine-item scale [30] and our previous publications [31] with minor modifications. In Item G, we considered the involvement of aged or female animals. Each item was given one point. Two reviewers independently evaluated the study quality. Divergences were well settled through consulting with correspondence authors. 


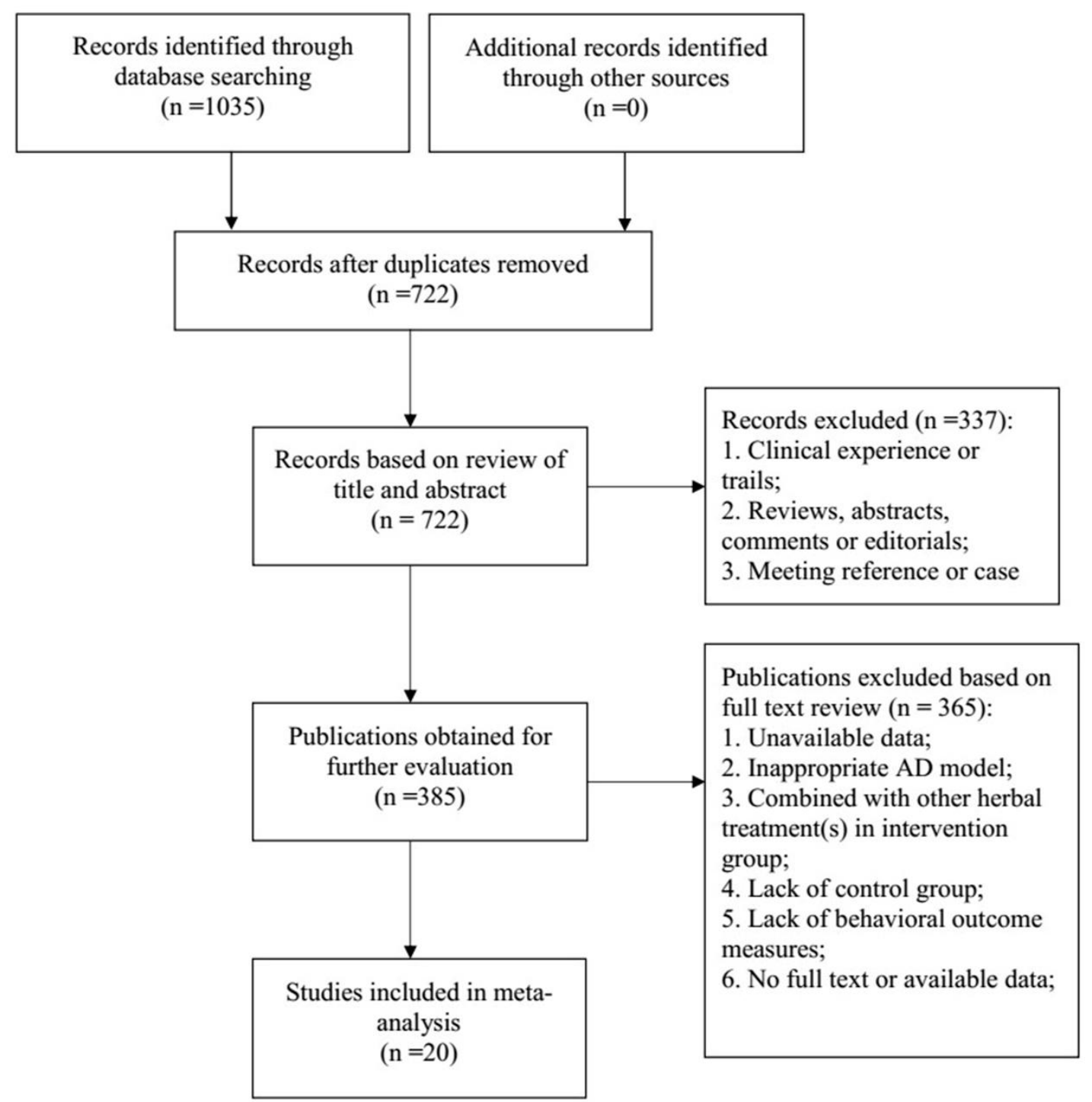

Figure 1. Summary of the process for identifying candidate studies.

\section{Statistical analysis}

The pooled analyses were carried out using RevMan 5.3 software. Heterogeneity across the subgroups was assessed using the Cochrane Q-statistic test and the $I^{2}$ statistic test. A fixed effects model $\left(I^{2}<50 \%\right)$ or a random effects model $\left(I^{2}>50 \%\right)$ was used depending on the value of $I^{2}$. We calculated the standard mean difference (SMD) with $95 \%$ confidence intervals (CIs). Sensitivity analyses omitting one study at a time from the original analysis were conducted to demonstrate our main results to be robust. Considering two-tailed statistical tests, results were considered statistically significant when $P<0.05$.

\section{RESULTS}

\section{Study selection}

A total of 1035 hits were found through the electrical database searching, of which 313 studies were duplicated. After screening titles and abstracts, 337 studies were excluded because they were clinical trials, case reports or review articles. Through full-text evaluation of the remaining 385 studies, 365 were excluded for at least one of the following reasons: (1) unavailable data; (2) inappropriate AD model; (3) combined with other herbal treatment(s) in the intervention group; (4) no control group; (5) no behavioral outcome measures. Eventually, 20 studies [32-51] were selected; see Fig. 1. 

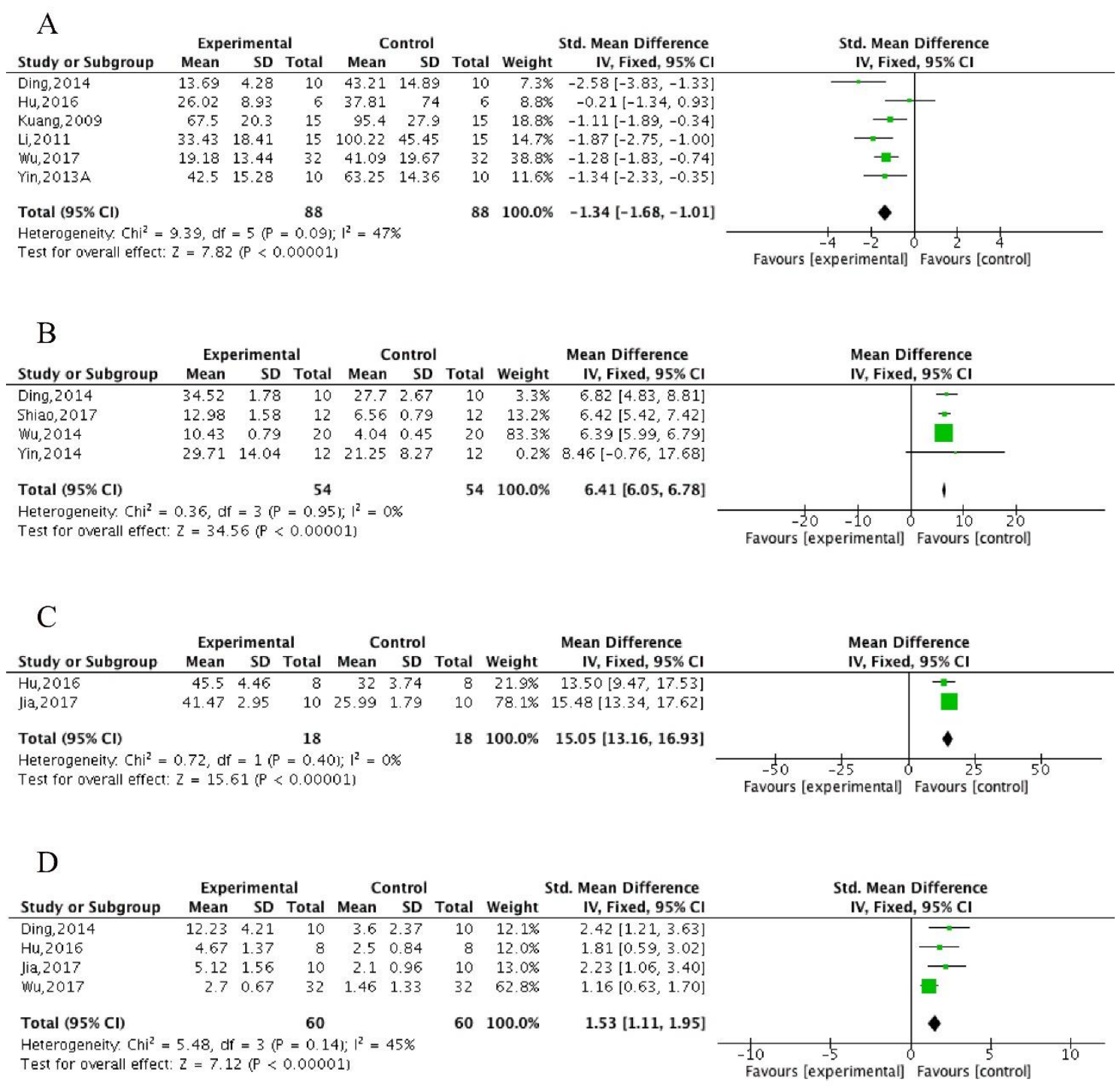

Figure 2. The forest plot in Morris water maze test. Effects of ECC for (A) decreasing the escape latency in spatial performance, increasing $(\mathbf{B})$ exact time/(C) percentage of time and $(\mathbf{D})$ increasing crossing numbers in platformquadrant in probe test compared with control group.

\section{Characteristics of included studies}

All studies were conducted in China and published between 2001 and 2017, of which 4 studies were published in English [33, 42, 45, 49]. In total, 1696 rats or mice were involved, and the sample size ranged from 40 to 192. A total of 6 different experimental rodent species were involved, including Kunming (KM) mouse $(\mathrm{n}=$ 1016, 59.91\%), senescence-accelerated mouseprone 8 (SAMP8) mouse $(\mathrm{n}=80,4.72 \%)$, NIH mouse $(\mathrm{n}=60$, $3.54 \%$ ), amyloid precursor protein/presenilin 1 (APP/PS1) transgenic mouse $(n=40,2.36 \%)$, Sprague-Dawley (SD) rat $(\mathrm{n}=440,25.94 \%)$ and Wistar rat $(\mathrm{n}=60,3.54 \%)$. Twelve studies used male rodents, 6 studies used both female and male rodents and the other 2 studies did not provide gender details. GCs were used in 13 studies, CDPS in 5 studies, AS in 4 studies, ECH in 2 studies and PhGs in 1 study. AD models were established by using $A \beta(1-42), A \beta(25-35)$ or $A \beta(1-40)$ cerebral ventricle infusion $(\mathrm{n}=7)$, using D-galactose (D-gal, $\mathrm{n}=6$ ), scopolamine $(n=6)$, sodium nitrite $(n=4)$, aluminium chloride $\left(\mathrm{AlCl}_{3}, \mathrm{n}=2\right)$ or quinolinic acid $(\mathrm{n}=1)$ intraperitoneal injection, or using SAMP8 mice $(n=2)$ and APP/PS1 transgenic mice $(\mathrm{n}=1)$ directly. The non- 
functional liquid/normal saline/no treatment control was introduced in all 20 studies; however, WCM control was introduced in 6studies, by donepezil [33, 47, 48] or huperzine A $[40,50,51]$. NFIs as primary outcome measures were carried out by the Morris water maze test $(\mathrm{n}=11)$, step-down test $(\mathrm{n}=10)$, electrical Y-maze test (n $=4)$, step-through test $(\mathrm{n}=3)$, open field test $(\mathrm{n}=2)$ and passage water maze test $(\mathrm{n}=1)$; see Table 1 .

Table 1. Characteristics of the included studies.

\begin{tabular}{|c|c|c|c|c|c|c|c|c|}
\hline $\begin{array}{l}\text { Study } \\
\text { (years) }\end{array}$ & $\begin{array}{l}\text { Type of } \\
\text { herbal or } \\
\text { bioactive } \\
\text { compound }\end{array}$ & $\begin{array}{l}\text { Species } \\
\text { Sex } \\
\text { Weight } \\
\text { N }\end{array}$ & Anesthetic & $\begin{array}{l}\text { Model } \\
\text { (method) }\end{array}$ & $\begin{array}{l}\text { Experimental } \\
\text { group }\end{array}$ & $\begin{array}{l}\text { Control } \\
\text { group }\end{array}$ & Outcome measure & $\begin{array}{l}\text { Intergroup } \\
\text { differences* }\end{array}$ \\
\hline \multirow[t]{3}{*}{$\begin{array}{l}\text { Kuang, } \\
2009\end{array}$} & $\mathrm{GCs}$ & $\begin{array}{l}\mathrm{KM} \\
\text { mice } \\
\mathrm{M} \\
18-22 \mathrm{~g} \\
75\end{array}$ & - & $\begin{array}{l}\text { D-gal and } \\
\text { sodium } \\
\text { nitrite }\end{array}$ & $\begin{array}{l}\text { GCs }(60,120 \\
\mathrm{mg} / \mathrm{kg}) \\
\text { ig, } 40 \sim 50 \mathrm{~d}\end{array}$ & $\begin{array}{l}\text { NS for } \\
\text { same } \\
\text { volume }\end{array}$ & $\begin{array}{l}\text { 1. Step-down test } \\
\text { 1.1.1 error number }(\mathrm{T}) \\
\text { 1.1.2 wrong react latency }(\mathrm{T}) \\
\text { 1.2.1 error number }(\mathrm{RT}) \\
\text { 1.2.2 wrong react latency }(\mathrm{RT}) \\
\text { 2. Step-through test } \\
\text { 2.1.1 error number }(\mathrm{T}) \\
\text { 2.1.2 latency }(\mathrm{T}) \\
\text { 2.2.1 error number }(\mathrm{RT}) \\
\text { 2.2.2 latency }(\mathrm{RT}) \\
\text { 3. Morris water maze test } \\
\text { escape latency }\end{array}$ & $\begin{array}{l}\text { 1.1.1 } \mathrm{P}<0.05 \\
\text { 1.1.2 } \mathrm{P}>0.05 \\
\text { 1.2.1 } \mathrm{P}<0.05 \\
\text { 1.2.2 } \mathrm{P}>0.05 \\
\\
\text { 2.1.1 } \mathrm{P}<0.05 \\
\text { 2.1.2 } \mathrm{P}>0.05 \\
\text { 2.2.1 } \mathrm{P}<0.05 \\
\text { 2.2.2 } \mathrm{P}<0.05 \\
\text { 3. } \mathrm{P}<0.01\end{array}$ \\
\hline & & $\begin{array}{l}\mathrm{KM} \\
\text { mice } \\
\mathrm{M} \\
18-22 \mathrm{~g} \\
50\end{array}$ & - & $\begin{array}{l}\text { D-gal and } \\
\text { sodium } \\
\text { nitrite }\end{array}$ & $\begin{array}{l}\text { GCs }(60,120 \\
\mathrm{mg} / \mathrm{kg}) \\
\text { ig, } 40 \sim 50 \mathrm{~d}\end{array}$ & $\begin{array}{l}\text { NS for } \\
\text { same } \\
\text { volume }\end{array}$ & $\begin{array}{l}\text { 4. Na+-K+ ATPase } \\
\text { 5. GSH-PX }\end{array}$ & $\begin{array}{l}\text { 4. } \mathrm{P}<0.05 \\
\text { 5. } \mathrm{P}<0.001\end{array}$ \\
\hline & & $\begin{array}{l}\text { SD rat } \\
\text { M } \\
180-200 \mathrm{~g} \\
40\end{array}$ & - & $\begin{array}{l}\text { D-gal and } \\
\text { sodium } \\
\text { nitrite }\end{array}$ & $\begin{array}{l}\text { GCs }(60,120 \\
\mathrm{mg} / \mathrm{kg}) \\
\text { ig, } 40 \sim 50 \mathrm{~d}\end{array}$ & $\begin{array}{l}\text { NS for } \\
\text { same } \\
\text { volume }\end{array}$ & $\begin{array}{l}\text { 6. SOD } \\
\text { 7. NO }\end{array}$ & $\begin{array}{l}\text { 6. } \mathrm{P}<0.001 \\
\text { 7. } \mathrm{P}<0.01\end{array}$ \\
\hline $\begin{array}{l}\text { Wu, } \\
2014\end{array}$ & GCs & $\begin{array}{l}\text { SD rats } \\
\mathrm{M} \\
300- \\
350 \mathrm{~g} \\
100\end{array}$ & phenobarbital & $\mathrm{A} \beta(1-42)$ & $\begin{array}{l}\text { GCs }(100, \\
200 \mathrm{mg} / \mathrm{kg}) \\
\mathrm{ig}, 7-14 \mathrm{~d}\end{array}$ & $\begin{array}{l}\text { a. sterile } \\
\text { distilled } \\
\text { water for } \\
\text { same } \\
\text { volume } \\
\text { b. } \\
\text { donepezil } \\
(0.75 \\
\mathrm{mg} / \mathrm{kg})\end{array}$ & $\begin{array}{l}\text { 1.Open field test } \\
\text { 1.1 time spend in the hole } \\
\text { 1.2 number of entries } \\
\text { 1.3 movement activity } \\
\text { 2.Step-through test } \\
\text { latency (T) } \\
\text { 3. Morris water maze test } \\
\text { 3.1 escape time } \\
\text { 3.2 exact time in platform-quadrant } \\
\text { 3.3 swimming velocity } \\
\text { 4. A } \beta \text { (1-42) deposition } \\
\text { 5. Neurotransmitters and } \\
\text { metabolites (ACh, NE, DA) } \\
\text { 6. Activity of AChE, MAO-A and } \\
\text { MAO-B }\end{array}$ & $\begin{array}{l}1.1 \mathrm{P}>0.05 \\
1.2 \mathrm{P}>0.05 \\
1.3 \mathrm{P}>0.05 \\
\text { 2. } \mathrm{P}<0.001 \\
\\
\text { 3.1 } \mathrm{P}<0.05 \\
\text { 3.2 } \mathrm{P}<0.001 \\
\text { 3.3 } \mathrm{P}>0.05 \\
\text { 4. } \mathrm{P}<0.01 \\
\text { 5. } \mathrm{P}<0.05 \\
\text { 6. } \mathrm{P}<0.05\end{array}$ \\
\hline $\begin{array}{l}\text { Liu, } \\
2005\end{array}$ & GCs & $\begin{array}{l}\text { KM } \\
\text { mice } \\
\text { M } \\
20-24 \mathrm{~g} \\
60\end{array}$ & $\begin{array}{l}\text { chloral } \\
\text { hydrate }\end{array}$ & $\begin{array}{l}\text { Quinolinic } \\
\text { acid }\end{array}$ & $\begin{array}{l}\text { GCs }(62.5, \\
125,250 \\
\mathrm{mg} / \mathrm{kg}) \\
\text { ig, 15d }\end{array}$ & $\begin{array}{l}\text { sterile } \\
\text { distilled } \\
\text { water for } \\
\text { same } \\
\text { volume }\end{array}$ & $\begin{array}{l}\text { 1.Step-down test } \\
\text { 1.1 error number (T) } \\
\text { 1.2 error number (RT) } \\
\text { 2. Electrical Y- maze test } \\
\text { right react times } \\
\text { 3. Activity of SOD, MDA and } \\
\text { GSH-PX. } \\
\text { 4. Neuron apoptosis } \\
\text { 5. Calcium content }\end{array}$ & $\begin{array}{l}\mathrm{P}<0.05 \\
\mathrm{P}<0.05 \\
\text { 2. } \mathrm{P}<0.05 \\
\text { 3. } \mathrm{P} \mathrm{P}<0.05 \\
\text { or } \mathrm{P}<0.01 \\
\text { 4. } \mathrm{P}<0.01 \\
\text { 5. } \mathrm{NG}\end{array}$ \\
\hline $\begin{array}{l}\text { Liu, } \\
2006\end{array}$ & GCs & $\begin{array}{l}\text { NIH } \\
\text { mice } \\
\mathrm{M} \\
20-24 \mathrm{~g} \\
60\end{array}$ & $\begin{array}{l}\text { chloral } \\
\text { hydrate }\end{array}$ & $\mathrm{A} \beta(25-35)$ & $\begin{array}{l}\text { GCs }(62.5, \\
125,250 \\
\mathrm{mg} / \mathrm{kg}) \\
\text { ig, 17d }\end{array}$ & $\begin{array}{l}\text { sterile } \\
\text { distilled } \\
\text { water for } \\
\text { same } \\
\text { volume }\end{array}$ & $\begin{array}{l}\text { 1. Step-down test } \\
\text { 1.1 error number (T) } \\
\text { 1.2 error number (RT) } \\
\text { 2. Activity of SOD, MDA and } \\
\text { GSH-PX } \\
\text { 3. Neuron apoptosis } \\
\text { 4. Bax / Bcl-2 }\end{array}$ & $\begin{array}{l}\mathrm{P}<0.01 \\
\mathrm{P}<0.01 \\
\text { 2. } \mathrm{P}<0.05 \text { or } \\
\mathrm{P}<0.01 \\
\text { 3. } \mathrm{P}<0.01 \\
\text { 4. } \mathrm{NG}\end{array}$ \\
\hline
\end{tabular}




\begin{tabular}{|c|c|c|c|c|c|c|c|c|}
\hline $\begin{array}{l}\text { Luo, } \\
2007\end{array}$ & GCs & $\begin{array}{l}\mathrm{KM} \\
\text { mice } \\
\mathrm{M} \\
20-24 \mathrm{~g} \\
60\end{array}$ & $\begin{array}{l}\text { chloral } \\
\text { hydrate }\end{array}$ & $\mathrm{AlCl} 3$ & $\begin{array}{l}\text { GCs }(62.5 \\
125,250 \\
\mathrm{mg} / \mathrm{kg}) \\
\text { ig, 20d }\end{array}$ & $\begin{array}{l}\text { NS for } \\
\text { same } \\
\text { volume }\end{array}$ & $\begin{array}{l}\text { 1. Step-down test } \\
\text { 1.1 error number }(\mathrm{T}) \\
\text { 1.2 wrong react latency }(\mathrm{T}) \\
\text { 2. Electrical Y-maze test } \\
\text { error react times } \\
\text { 3. Activity of SOD and MDA } \\
\text { 4. Brain weight coefficient }\end{array}$ & $\begin{array}{l}\text { 1.1 } \mathrm{P}<0.05 \\
1.2 \mathrm{P}<0.05 \\
\text { 2. } \mathrm{P}<0.01 \\
\text { 3. } \mathrm{P}<0.05 \text { or } \\
\mathrm{P}<0.01 \\
\text { 4. } \mathrm{P}<0.01\end{array}$ \\
\hline $\begin{array}{l}\text { Luo, } \\
2013\end{array}$ & GCs & $\begin{array}{l}\text { SD rats } \\
\mathrm{M} \\
220- \\
270 \mathrm{~g} \\
60\end{array}$ & $\begin{array}{l}\text { chloral } \\
\text { hydrate }\end{array}$ & $\mathrm{A} \beta(25-35)$ & $\begin{array}{l}\text { GCs }(40,80, \\
120 \mathrm{mg} / \mathrm{kg}) \\
\text { ig, } 14 \mathrm{~d}\end{array}$ & $\begin{array}{l}\text { NS for } \\
\text { same } \\
\text { volume }\end{array}$ & $\begin{array}{l}\text { 1. Step-down test } \\
\text { 1.1 error number }(\mathrm{T}) \\
\text { 1.2 reaction time }(\mathrm{T}) \\
\text { 2. Electrical Y-maze test } \\
\text { error react times } \\
\text { 3. Activity of AchE } \\
\text { 4. Calcium content }\end{array}$ & $\begin{array}{l}\text { 1.1 } \mathrm{P}<0.01 \\
\text { 1.2 } \mathrm{P}<0.01 \\
\text { 2. } \mathrm{P}<0.01 \\
\text { 3. } \mathrm{P}<0.01 \\
\text { 4. } \mathrm{NG}\end{array}$ \\
\hline $\begin{array}{l}\text { Yin, } \\
\text { 2013(A) }\end{array}$ & CDPS & $\begin{array}{l}\text { SD rats } \\
\mathrm{M} / \mathrm{F} \\
200- \\
250 \mathrm{~g} \\
60\end{array}$ & $\begin{array}{l}\text { chloral } \\
\text { hydrate }\end{array}$ & $\mathrm{A} \beta(25-35)$ & $\begin{array}{l}\text { CDPS }(20, \\
40,80 \mathrm{mg} / \mathrm{kg}) \\
\text { ig, } 28 \mathrm{~d}\end{array}$ & $\begin{array}{l}\text { NS for } \\
\text { same } \\
\text { volume }\end{array}$ & $\begin{array}{l}\text { 1. Morris water maze test } \\
\text { escape latency } \\
\text { 2. Neuron apoptosis } \\
\text { 3. Expression of Bcl-2 and caspase-3 }\end{array}$ & $\begin{array}{l}\text { 1. } \mathrm{P}<0.01 \\
\text { 2. } \mathrm{P}<0.01 \\
\text { 3. } \mathrm{P}<0.01\end{array}$ \\
\hline $\begin{array}{l}\text { Yin, } \\
\text { 2013(B) }\end{array}$ & CDPS & $\begin{array}{l}\text { Wistar } \\
\text { rats } \\
\text { NG } 180- \\
220 g \\
60\end{array}$ & $\begin{array}{l}\text { chloral } \\
\text { hydrate }\end{array}$ & $\mathrm{A} \beta(1-40)$ & $\begin{array}{l}\text { CDPS (L, M, } \\
\text { H) } \\
\text { ig, 28d }\end{array}$ & $\begin{array}{l}\text { corn oil } \\
\text { for same } \\
\text { volume }\end{array}$ & $\begin{array}{l}\text { 1. Morris water maze test } \\
\text { escape latency } \\
\text { 2. Activity of SOD and MDA } \\
\text { 3. Activity of NO, ONOO- and } \\
\text { ROS }\end{array}$ & $\begin{array}{l}\text { 1. } \mathrm{P}<0.01 \\
\text { 2. } \mathrm{P} \mathrm{P}<0.05 \\
\text { or } \mathrm{P}<0.01 \\
\text { 3. } \mathrm{P}<0.05 \text { or } \\
\mathrm{P}<0.01\end{array}$ \\
\hline $\mathrm{Li}, 2011$ & CDPS & $\begin{array}{l}\mathrm{KM} \\
\text { mice } \\
\mathrm{M} / \mathrm{F} \\
18-22 \mathrm{~g} \\
75\end{array}$ & - & Scopolamine & $\begin{array}{l}\text { CDPS (10, } \\
20,60 \mathrm{mg} / \mathrm{kg}) \\
\text { NG }\end{array}$ & $\begin{array}{l}\text { NS for } \\
\text { same } \\
\text { volume }\end{array}$ & $\begin{array}{l}\text { 1. Passage water maze test } \\
\text { 1.1 error number }(\mathrm{T}) \\
1.2 \text { latency }(\mathrm{T}) \\
\text { 2. Morris water maze test } \\
\text { escape latency } \\
\text { 3. Activity of SOD, MDA and } \\
\text { AChE }\end{array}$ & $\begin{array}{l}\mathrm{P}<0.01 \\
\mathrm{P}<0.01 \\
\text { 2. } \mathrm{P}<0.05 \\
\text { 3. } \mathrm{P}<0.05 \text { or } \\
\mathrm{P}<0.01\end{array}$ \\
\hline $\begin{array}{l}\text { Ding, } \\
2014\end{array}$ & $\mathrm{ECH}$ & $\begin{array}{l}\text { SD rats } \\
\text { M } \\
290- \\
320 \mathrm{~g} \\
60\end{array}$ & $\begin{array}{l}\text { chloral } \\
\text { hydrate }\end{array}$ & $\begin{array}{l}\text { D-gal and } \\
A \beta(25-35)\end{array}$ & $\begin{array}{l}\text { ECH }(10,20, \\
40 \mathrm{mg} / \mathrm{kg}) \\
\text { ig, } 28 \mathrm{~d}\end{array}$ & $\begin{array}{l}\text { a. NS for } \\
\text { same } \\
\text { volume } \\
\text { b. } \\
\text { huperzine- } \\
\text { A }(0.02 \\
\mathrm{mg} / \mathrm{kg})\end{array}$ & $\begin{array}{l}\text { 1. Morris water maze test } \\
\text { 1.1 escape latency } \\
1.2 \text { number of platform crossing } \\
\text { 1.3 exact time in platform-quadrant } \\
\text { 2. Activity of NE, DA and 5-TH }\end{array}$ & $\begin{array}{l}\text { 1.1 } \mathrm{P}<0.01 \\
1.2 \mathrm{P}<0.01 \\
1.3 \mathrm{NG} \\
\text { 2. } \mathrm{P}<0.05\end{array}$ \\
\hline $\begin{array}{l}\text { Peng, } \\
2014\end{array}$ & AS & $\begin{array}{l}\mathrm{KM} \\
\text { mice } \\
\mathrm{F} \\
16-20 \mathrm{~g} \\
120\end{array}$ & - & $\begin{array}{l}\text { D-gal and } \\
\mathrm{AlCl} 3\end{array}$ & $\begin{array}{l}\text { AS }(30,60 \\
120 \mathrm{mg} / \mathrm{kg}) \\
\mathrm{ig}, 30 \mathrm{~d}\end{array}$ & $\begin{array}{l}\mathrm{NS}(10 \\
\mathrm{ml} / \mathrm{kg})\end{array}$ & $\begin{array}{l}\text { 1. Step-down test } \\
\text { 1.1.1 error number }(\mathrm{T}) \\
\text { 1.1.2 wrong react latency }(\mathrm{T}) \\
\text { 1.2.1 error number }(\mathrm{RT}) \\
\text { 1.2.2 wrong react latency }(\mathrm{RT}) \\
\text { 2. Level of NO } \\
\text { 3.Pathomorphological changes in } \\
\text { the hippocampus } \\
\text { 4. Expression of Caspase-3 }\end{array}$ & $\begin{array}{l}\text { 1.1.1 } \mathrm{P}<0.01 \\
\text { 1.1.2 } \mathrm{P}>0.05 \\
\text { 1.2.1 } \mathrm{P}<0.01 \\
\text { 1.2.2 } \mathrm{P}>0.01 \\
\text { 2. } \mathrm{P}<0.01 \\
\text { 3. } \mathrm{NG} \\
\text { 4. } \mathrm{P}<0.05\end{array}$ \\
\hline $\begin{array}{l}\mathrm{Hu}, \\
2016\end{array}$ & AS & $\begin{array}{l}\text { APP/PSI } \\
\text { mice } \\
\text { NG } \\
25-35 \mathrm{~g} \\
40\end{array}$ & - & - & $\begin{array}{l}\text { AS }(30,60 \\
120 \mathrm{mg} / \mathrm{kg}) \\
\mathrm{ig}, 60 \mathrm{~d}\end{array}$ & $\begin{array}{l}\text { sterile } \\
\text { distilled } \\
\text { water for } \\
\text { same } \\
\text { volume }\end{array}$ & $\begin{array}{l}\text { 1.Morris water maze test } \\
\text { 1.1 escape latency } \\
\text { 1.2 number of platform crossing } \\
\text { 1.3 percentage of time in platform- } \\
\text { quadrant } \\
\text { 2. Neuron apoptosis } \\
\text { 3. Survival neuron number } \\
\text { 4. A } \beta(1-42) \text { deposition }\end{array}$ & $\begin{array}{l}\text { 1. } 1 \mathrm{P}<0.01 \\
1.2 \mathrm{P}<0.05 \\
1.3 \mathrm{P}<0.01 \\
\text { 2. } \mathrm{P}<0.05 \\
\text { 3. } \mathrm{P}<0.05 \\
\text { 4. } \mathrm{P}<0.05\end{array}$ \\
\hline $\begin{array}{l}\text { Jia, } \\
2014\end{array}$ & GCs & $\begin{array}{l}10- \\
\text { month- } \\
\text { old } \\
\text { SAMP8 } \\
\text { mice } \\
\text { M } \\
25-35 \mathrm{~g} \\
40\end{array}$ & - & - & $\begin{array}{l}\text { GCs }(100 \mathrm{mg}) \\
\text { ig, } 30 \mathrm{~d}\end{array}$ & $\begin{array}{l}\text { NS for } \\
\text { same } \\
\text { volume }\end{array}$ & $\begin{array}{l}\text { 1. Morris water maze test } \\
\text { 1.1 escape latency } \\
\text { 1.2 number of platform crossing } \\
1.3 \text { time in the target quadrant } \\
\text { 1.4 swimming speed } \\
\text { 2. Survival neuron number } \\
\text { 3. Activity of MDA, SOD and } \\
\text { GSH-PX }\end{array}$ & $\begin{array}{l}\text { 1. } 1 \mathrm{P}<0.01 \\
1.2 \mathrm{P}<0.01 \\
1.3 \mathrm{P}<0.01 \\
1.4 \mathrm{P}>0.05 \\
\text { 2. } \mathrm{P}<0.01 \\
\text { 3. } \mathrm{P}<0.05 \text { or } \\
\mathrm{P}<0.01\end{array}$ \\
\hline
\end{tabular}




\begin{tabular}{|c|c|c|c|c|c|c|c|c|}
\hline $\begin{array}{l}\text { Jia, } \\
2017\end{array}$ & PhG & $\begin{array}{l}10- \\
\text { month- } \\
\text { old } \\
\text { SAMP8 } \\
\text { mice } \\
\text { M } \\
30 \mathrm{~g} \\
40\end{array}$ & - & - & $\begin{array}{l}\mathrm{PhG}(25,50, \\
100 \mathrm{mg} / \mathrm{kg}) \\
\mathrm{ig}, 30 \mathrm{~d}\end{array}$ & $\begin{array}{l}\text { NS for } \\
\text { same } \\
\text { volume }\end{array}$ & $\begin{array}{l}\text { 1. Morris water maze test } \\
1.1 \text { escape latency } \\
1.2 \text { number of platform crossing } \\
1.3 \text { percentage of time in platform- } \\
\text { quadrant } \\
1.4 \text { path length } \\
\text { 2. Activity of MDA, SOD and } \\
\text { GSH-PX } \\
\text { 3.Density of dendritic spines } \\
\text { 4.Expression of SYN and PSD-95 }\end{array}$ & $\begin{array}{l}\mathrm{P}<0.05 \\
\mathrm{P}<0.05 \\
\mathrm{P}<0.05 \\
\mathrm{P}<0.05 \\
\text { 2. } \mathrm{P}<0.05 \text { or } \\
\mathrm{P}<0.01 \\
\text { 3. } \mathrm{P}<0.05 \\
\text { 4. } \mathrm{P}<0.05\end{array}$ \\
\hline $\begin{array}{l}\text { Gao, } \\
2005\end{array}$ & GCs & $\begin{array}{l}\mathrm{KM} \\
\mathrm{mice} \\
\mathrm{M} / \mathrm{F} \\
18-22 \mathrm{~g} \\
180 \\
\end{array}$ & - & Scopolamine & $\begin{array}{l}\text { GCs (L, M, } \\
\text { H) } \\
\text { ig, 30d }\end{array}$ & $\begin{array}{l}\text { sterile } \\
\text { distilled } \\
\text { water for } \\
\text { same } \\
\text { volume } \\
\end{array}$ & $\begin{array}{l}\text { 1. Step-down test } \\
1.1 \text { error number }(\mathrm{T}) \\
1.2 \text { wrong react latency }(\mathrm{T})\end{array}$ & $\begin{array}{l}\mathrm{P}<0.01 \\
1.2 \mathrm{P}<0.01\end{array}$ \\
\hline $\begin{array}{l}\mathrm{Wu}, \\
2017\end{array}$ & CDPS & $\begin{array}{l}\mathrm{KM} \\
\mathrm{mice} \\
\mathrm{M} / \mathrm{F} \\
18-22 \mathrm{~g} \\
192 \\
\end{array}$ & - & D-gal & $\begin{array}{l}\text { CDPS (25, } \\
50,100 \\
\mathrm{mg} / \mathrm{kg}) \\
\mathrm{ig}, 42 \mathrm{~d}\end{array}$ & $\begin{array}{l}\text { NS for } \\
\text { same } \\
\text { volume }\end{array}$ & $\begin{array}{l}\text { 1. Morris water maze test } \\
1.1 \text { escape latency } \\
1.2 \text { number of platform crossing }\end{array}$ & $\begin{array}{l}\mathrm{P}<0.05 \\
\mathrm{P}<0.05\end{array}$ \\
\hline $\begin{array}{l}\text { Yin, } \\
2014\end{array}$ & CDPS & $\begin{array}{l}\mathrm{KM} \\
\text { mice } \\
\mathrm{M} / \mathrm{F} \\
23-27 \mathrm{~g} \\
72\end{array}$ & - & Scopolamine & $\begin{array}{l}\text { CDPS (25, } \\
50,100 \\
\mathrm{mg} / \mathrm{kg}) \\
\mathrm{ig}, 42 \mathrm{~d}\end{array}$ & $\begin{array}{l}\text { a. sterile } \\
\text { distilled } \\
\text { water for } \\
\text { same } \\
\text { volume } \\
\text { b. } \\
\text { donepezil } \\
(0.8 \\
\mathrm{mg} / \mathrm{kg})\end{array}$ & $\begin{array}{l}\text { Morris water maze test } \\
1.1 \text { escape latency } \\
1.2 \text { exact time in platform-quadrant } \\
1.3 \text { path length } \\
\text { 2. Step-down test } \\
\text { 2.1.1 error number (T) } \\
\text { 2.1.2 right latency (T) } \\
\text { 2.2.1 error number (RT) } \\
\text { 2.2.2 right latency (RT) } \\
\text { 3. Expression of GAP- } 43 \text { and SYP } \\
\text { 4. Number and morphology of } \\
\text { synapses }\end{array}$ & $\begin{array}{l}1.1 \mathrm{NG} \\
1.2 \mathrm{P}<0.05 \\
1.3 \mathrm{NG} \\
\\
\text { 2.1.1 } \mathrm{P}<0.05 \\
\text { 2.1.2 } \mathrm{P}>0.05 \\
\text { 2.2.1 } \mathrm{P}<0.05 \\
\text { 2.2.2 } \mathrm{P}<0.05 \\
\text { 3. } \mathrm{P}<0.05 \\
\text { 4. } \mathrm{P}<0.05\end{array}$ \\
\hline $\begin{array}{l}\text { Shiao, } \\
2017\end{array}$ & $\mathrm{ECH}$ & $\begin{array}{l}\text { SD rat } \\
\mathrm{M} \\
300-350 \\
120\end{array}$ & phenobarbital & $\begin{array}{l}\mathrm{A} \beta(1-42) / \\
\text { Scopolamine }\end{array}$ & $\begin{array}{l}\mathrm{ECH}(2.5,5.0 \\
\mathrm{mg} / \mathrm{kg}) \\
\mathrm{ig}, 15 \mathrm{~d}\end{array}$ & $\begin{array}{l}\text { a. sterile } \\
\text { distilled } \\
\text { water for } \\
\text { same } \\
\text { volume } \\
\text { b. } \\
\text { donepezil } \\
(0.75 \\
\mathrm{mg} / \mathrm{kg})\end{array}$ & $\begin{array}{l}\text { 1. Open-field task } \\
\text { 1.1 time spend in the hole } \\
1.2 \text { number of entries into the hole } \\
\text { 1.3 movement activity } \\
\text { 2. Step-through test } \\
\text { 2.1 latency (T) } \\
\text { 2.2 latency (RT) } \\
\text { 3. Morris water maze test } \\
\text { 3.1 escape latency } \\
\text { 3.2 exact time in platform-quadrant } \\
\text { 3.3 swimming velocity } \\
\text { 4. A }(1-42) \text { deposition } \\
\text { 5. Levels of Ach, NE and DA } \\
\text { 6. Activity of AChE, MAO-A and } \\
\text { MAO -B }\end{array}$ & $\begin{array}{l}1.1 \mathrm{P}<0.05 \\
1.2 \mathrm{P}<0.05 \\
1.3 \mathrm{P}>0.05 \\
2.1 \mathrm{P}<0.05 \\
2.2 \mathrm{P}>0.05 \\
3.1 \mathrm{P}<0.05 \\
3.2 \mathrm{P}<0.05 \\
3.3 \mathrm{P}>0.05 \\
4 . \mathrm{P}<0.05 \\
\text { 5. } \mathrm{P}<0.05 \text { or } \\
\mathrm{P}<0.01 \\
6 . \mathrm{P}<0.05 \text { or } \\
\mathrm{P}<0.01\end{array}$ \\
\hline $\begin{array}{l}\text { Piao, } \\
2001\end{array}$ & AS & $\begin{array}{l}\mathrm{KM} \\
\text { mice } \\
\mathrm{M} \\
18-22 \\
60\end{array}$ & - & Scopolamine & $\begin{array}{l}\mathrm{AS}(5,10 \\
\mathrm{mg} / \mathrm{kg}) \\
\mathrm{ig}, 10 \mathrm{~d}\end{array}$ & $\begin{array}{l}\text { a. NS for } \\
\text { same } \\
\text { volume } \\
\text { b. } \\
\text { huperzine- } \\
\text { A }(0.07 \\
\mathrm{mg} / \mathrm{kg})\end{array}$ & $\begin{array}{l}\text { 1. Step-down test } \\
\text { 1.1.1 wrong react latency }(\mathrm{T}) \\
\text { 1.1.2 error time }(\mathrm{T}) \\
\text { 1.2.1 wrong react latency }(\mathrm{RT}) \\
\text { 1.2.2 error time }(\mathrm{RT}) \\
\text { 2. Electrical } \mathrm{Y} \text {-maze test } \\
\text { right react times } \\
\text { 3. Activity of AChE } \\
\end{array}$ & $\begin{array}{l}\text { 1.1.1 NG } \\
1.1 .2 \mathrm{P}>0.05 \\
1.2 .1 \mathrm{P}<0.05 \\
\text { 1.2.2 } \mathrm{P}<0.05 \\
\text { 2. } \mathrm{P}<0.05 \\
\text { 3. } \mathrm{P}<0.05\end{array}$ \\
\hline $\begin{array}{l}\text { Lin, } \\
2012\end{array}$ & AS & $\begin{array}{l}\mathrm{KM} \\
\text { mice } \\
\mathrm{M} \\
18-22 \mathrm{~g} \\
72\end{array}$ & - & Scopolamine & $\begin{array}{l}\text { AS }(30,60, \\
120 \mathrm{mg} / \mathrm{kg}) \\
\text { ig, } 10 \mathrm{~d}\end{array}$ & $\begin{array}{l}\text { a. NS for } \\
\text { same } \\
\text { volume } \\
\text { b. } \\
\text { huperzine- } \\
\text { A }(0.07 \\
\mathrm{mg} / \mathrm{kg})\end{array}$ & $\begin{array}{l}\text { 1. Step-down test } \\
\text { 1.1.1 error number (T) } \\
\text { 1.1.2 wrong react latency (T) } \\
\text { 1.2.1 error number (RT) } \\
\text { 1.2.2 wrong react latency (RT) } \\
\text { 2. Activity of MDA, SOD and } \\
\text { GSH-PX } \\
\text { 3. Protein content in brain tissue }\end{array}$ & $\begin{array}{l}1.1 .1 \mathrm{NG} \\
1.1 .2 \mathrm{NG} \\
1.2 .1 \mathrm{P}<0.01 \\
1.2 .2 \mathrm{P}<0.01 \\
2 . \mathrm{P}<0.05 \text { or } \\
\mathrm{P}<0.01 \\
\text { 3. } \mathrm{P}>0.05\end{array}$ \\
\hline
\end{tabular}

Note. GCs: glycosides of Cictanches. CDPS: polysacchrides of Cistanches deserticola. ECH: echinacoside. AS: acteoside. PhGs: phenylethanoid glycosides. KM mice: Kunming mice. SD rats: Sprague-Dawley rats. NIH mice: National Institutes of Health mice. SAMP8 mice: senescence-accelerated mouseprone 8 mice. APP/PS1 mice: amyloid precursor protein/presenilin 1 transgenic mice. M: male. F: female. -: no. D-gal: D-galactose. NG, not given. A 3 : amyloid $\beta$. AlCl ${ }_{3}$ : aluminium chloride. ig: intragastric administration. L: low dose. M: medium dose. H: high dose. d: day. NS: normal saline. T: in the training test. RT: in the retention test. $\mathrm{Na}^{+}-\mathrm{K}^{+}$ATPase: sodium-potassium adenosine triphosphatase. GSH-PX: glutathione peroxidase. SOD: superoxide dismutase. NO: nitric oxide. AChE: acetylcholinesterase. ACh: acetylcholine. NE: norepinephrine. DA: dopamine. MDA: malondialdehyde. 5-TH: 5-hydroxytryptamine. MAO-A: monoamine oxidase A. MAO-B: monoamine oxidase B. Bax: B-cell lymphoma/leukemia-2 associated X protein. Bcl-2: B-cell lymphoma/leukemia-2. *: the intergroup differences listed were ECC vs. negative control group (NS or sterile distilled water), the intergroup differences of ECC vs. modern western conventional treatments were not given. 

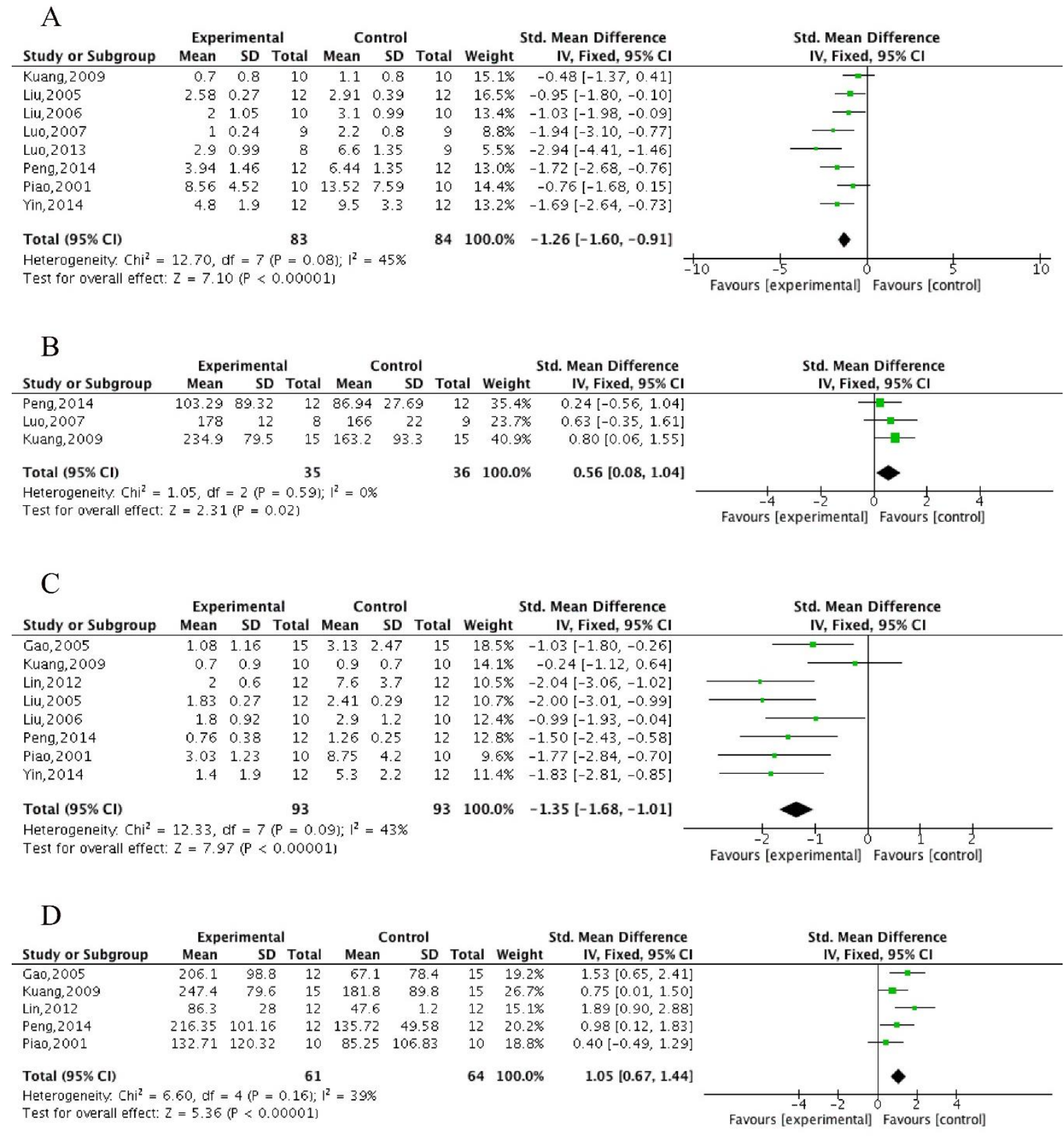

Figure 3. The forest plot in Step-down test. Effects of ECC for decreasing (A) error times and (B) wrong react latency in training test and decreasing $(\mathbf{C})$ error times and $(\mathbf{D})$ wrong react latency in retention test compared with control group.

\section{Study quality}

The quality of the 20 included studies ranged from 4 to 7 , with a mean score of 5.05. All studies used random allocation and declared no potential conflict of interests. Nineteen studies were peer reviewed, while one study [32] was an online $\mathrm{PhD}$ thesis. The use of anesthetic without significant intrinsic neuroprotective activity was reported in 18 studies, compliance with animal welfare regulations in 10 studies, control of temperature in 8 studies and animal models with relevant comorbidities in 8 studies. No study reported sample size calculation or blinded assessment of the model or outcome; see Table 2. 

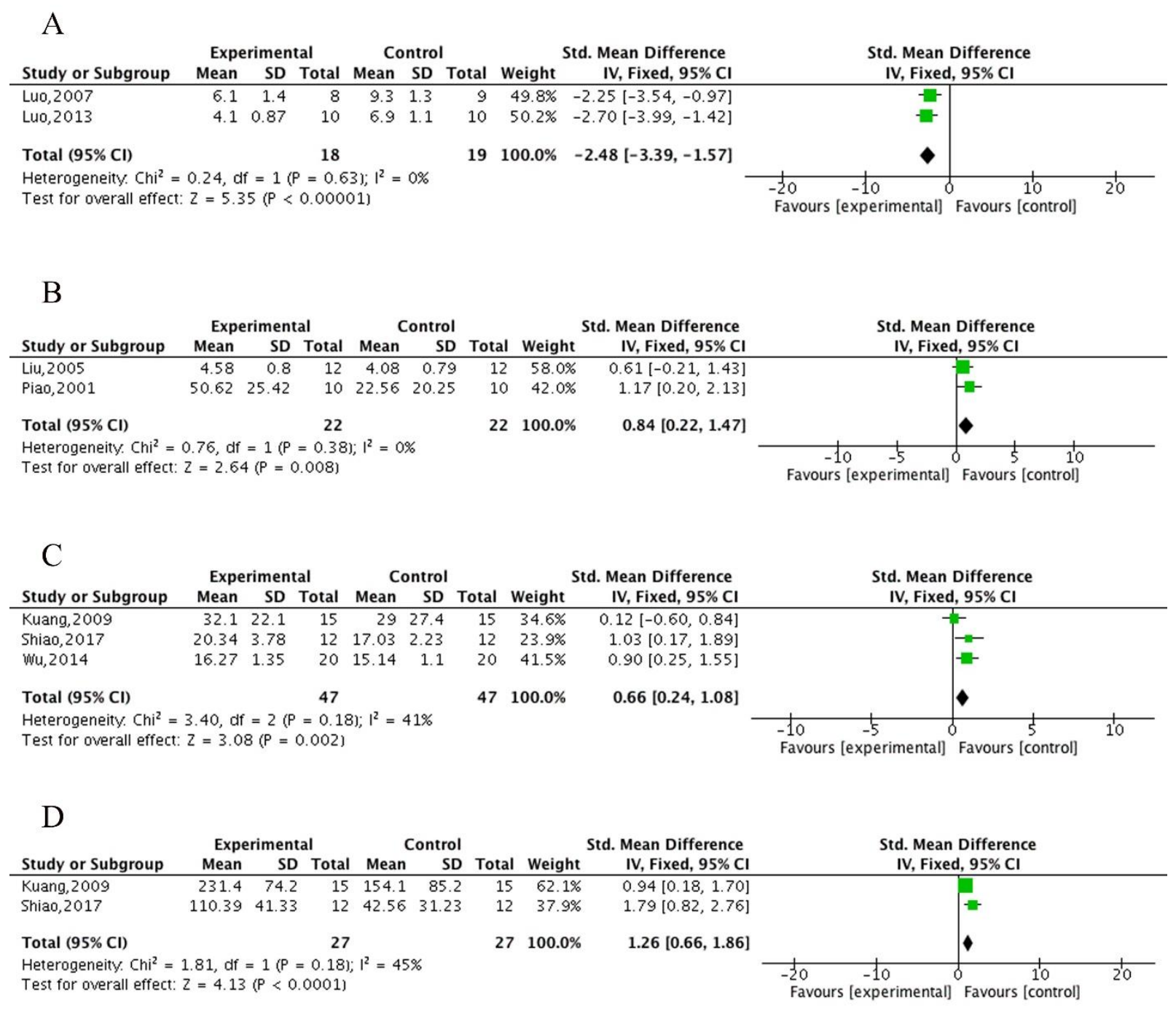

Figure 4. The forest plot in Electrical Y-maze test and Step-through test. Effects of ECC for (A) decreasing error react times, (B) increasing right react times in Electrical Y-maze test, and decreasing latency in training test (C) / retention test (D) in Step-through test compared with control group.

\section{Effectiveness}

\section{Neurobehavioral function indices}

The Morris water maze test, including the spatial test and the probe test, was conducted in 11 studies [32, 33, 3841, 43, 45, 47-49]. All 11 studies reported the spatial test using the escape latency as an outcome measure, of which 3 studies $[33,48,49]$ provided graphic data, and we failed to apply digital ruler software or to get in touch with the author for further information. Meta-analysis of 8 studies showed ECC significantly decreased the escape latency compared with the control $(\mathrm{n}=216, \mathrm{MD}=-1.46,95 \% \mathrm{CI}$
[ -1.79 to -1.12$], P<0.00001$; heterogeneity: $\chi^{2}=58.87$, df $\left.=7(P<0.00001) ; I^{2}=88 \%\right)$. Owing to obvious heterogeneity, we used sensitivity analyses and removed the respective outlier studies. Meta-analysis of 6 studies $[32,38,40,41,43,47]$ showed a significant effect of ECC in decreasing the escape latency in spatial performance compared with control $(\mathrm{n}=176, \mathrm{MD}=-1.34,95 \% \mathrm{CI}$ [ -1.68 to -1.01$], P<0.00001$; heterogeneity: $\chi^{2}=9.39$, df $=5(P=0.09) ; I^{2}=47 \%$; Fig. 2A). Four studies [33, 41, $48,49]$ showed an insignificant decrease in escape latency in ECC group compared with WCM control; however, meta-analysis was failed owing to unavailable data in 3 studies [33, 48, 49]. In the probe test, meta-analysis of 4 
studies [33, 41, 48, 49] showed ECC were significant for increasing exact time in platform-quadrant $(\mathrm{n}=108, \mathrm{MD}$ $=6.41,95 \% \mathrm{CI}$ [6.05 to 6.78], $P<0.00001$; heterogeneity: $\chi^{2}=0.36, \mathrm{df}=3(P=0.95) ; I^{2}=0 \%$; Fig. 2B $), 2$ studies $[43,45]$ for increasing percentage of time in the platformquadrant $(\mathrm{n}=36, \mathrm{MD}=15.05,95 \% \mathrm{CI}$ [13.16 to 16.93 ], $P<0.00001$; heterogeneity: $\chi^{2}=0.72, \mathrm{df}=1(P=0.400) ; I^{2}$ $=0 \%$; Fig. $2 \mathrm{C})$ and 4 studies [41, 43, 45, 47] for increasing number of platform crossings $(\mathrm{n}=120, \mathrm{MD}=1.53$, 95\%CI [1.11 to 1.95], $P<0.00001$; heterogeneity: $\chi^{2}=$ $5.48, \mathrm{df}=3(P=0.14) ; I^{2}=45 \%$; Fig. 2D) compared with controls. Meta-analysis of 4 studies [33, 41, 48, 49] showed there were no significant intergroup differences between the ECC group and WCM controls in increasing the exact time in the platform-quadrant $(\mathrm{n}=108, \mathrm{MD}=$ $0.81,95 \%$ CI [0.35 to 1.27$], P=0.06$; heterogeneity: $\chi^{2}=$ 18.62 , df $\left.=3(P=0.03) ; I^{2}=84 \%\right)$. We carried out a sensitive analysis by removing one study [49] of an obviously less effective ECC dose and markedly reduced the heterogeneity $(\mathrm{n}=84, \mathrm{MD}=0.46,95 \% \mathrm{CI}[-0.03$ to 0.94], $P=0.07$; heterogeneity: $\chi^{2}=0.94, \mathrm{df}=3(P=0.63)$; $I^{2}=0 \%$; Supplementary Fig. 1A). Two studies $[45,48]$ showed ECC significantly decreased the total swimming length $(P<0.05)$, whereas two other studies [33, 49] showed there were no significant difference in reducing the swimming velocity $(P>0.05)$ compared with controls.

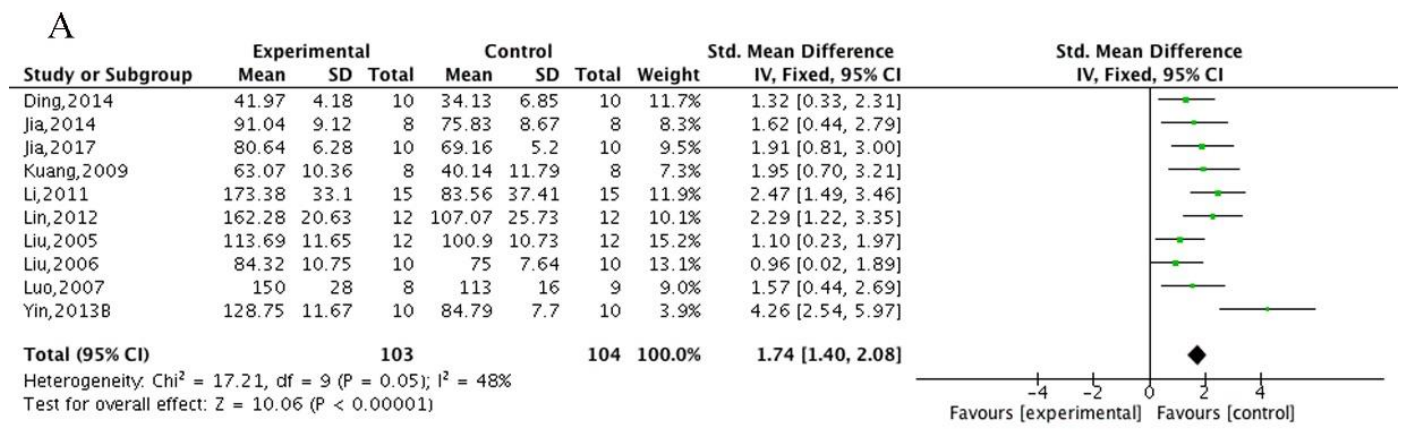

\begin{tabular}{|c|c|c|c|c|c|}
\hline \multirow[b]{2}{*}{ Study or Subgroup } & \multicolumn{3}{|c|}{ Experimental } & \multicolumn{2}{|c|}{ Control } \\
\hline & Mean & SD & Total & Mean & SD \\
\hline Ding, 2014 & 3.99 & 0.25 & 10 & 4.4 & 0.17 \\
\hline Jia, 2014 & 10.86 & 2.95 & 8 & 16.34 & 3.77 \\
\hline Jia, 2017 & 9.94 & 1.65 & 10 & 14.32 & 2.23 \\
\hline $\mathrm{Li}, 2011$ & 15.37 & 3.43 & 15 & 29.43 & 8.76 \\
\hline Lin, 2012 & 2.73 & 0.41 & 12 & 3.11 & 0.47 \\
\hline Liu, 2005 & 16.61 & 2.48 & 12 & 19.05 & 2.64 \\
\hline Liu, 2006 & 12.65 & 3.11 & 10 & 16 & 3.46 \\
\hline Luo, 2007 & 14 & 8 & 8 & 30 & 7 \\
\hline Yin, $2013 \mathrm{~B}$ & 8.67 & 2.67 & 8 & 20.53 & 3.34 \\
\hline Total $(95 \% \mathrm{Cl})$ & & & 93 & & \\
\hline
\end{tabular}

Test for overall effect: $z=8.69(P<0.00001)$

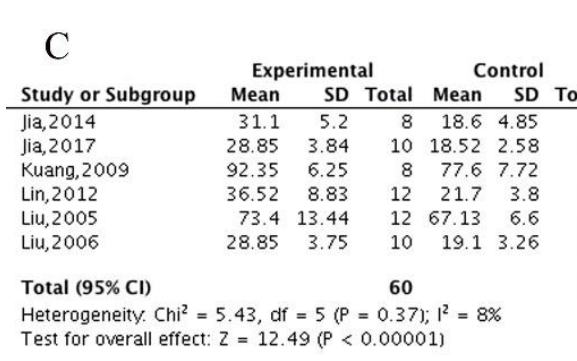
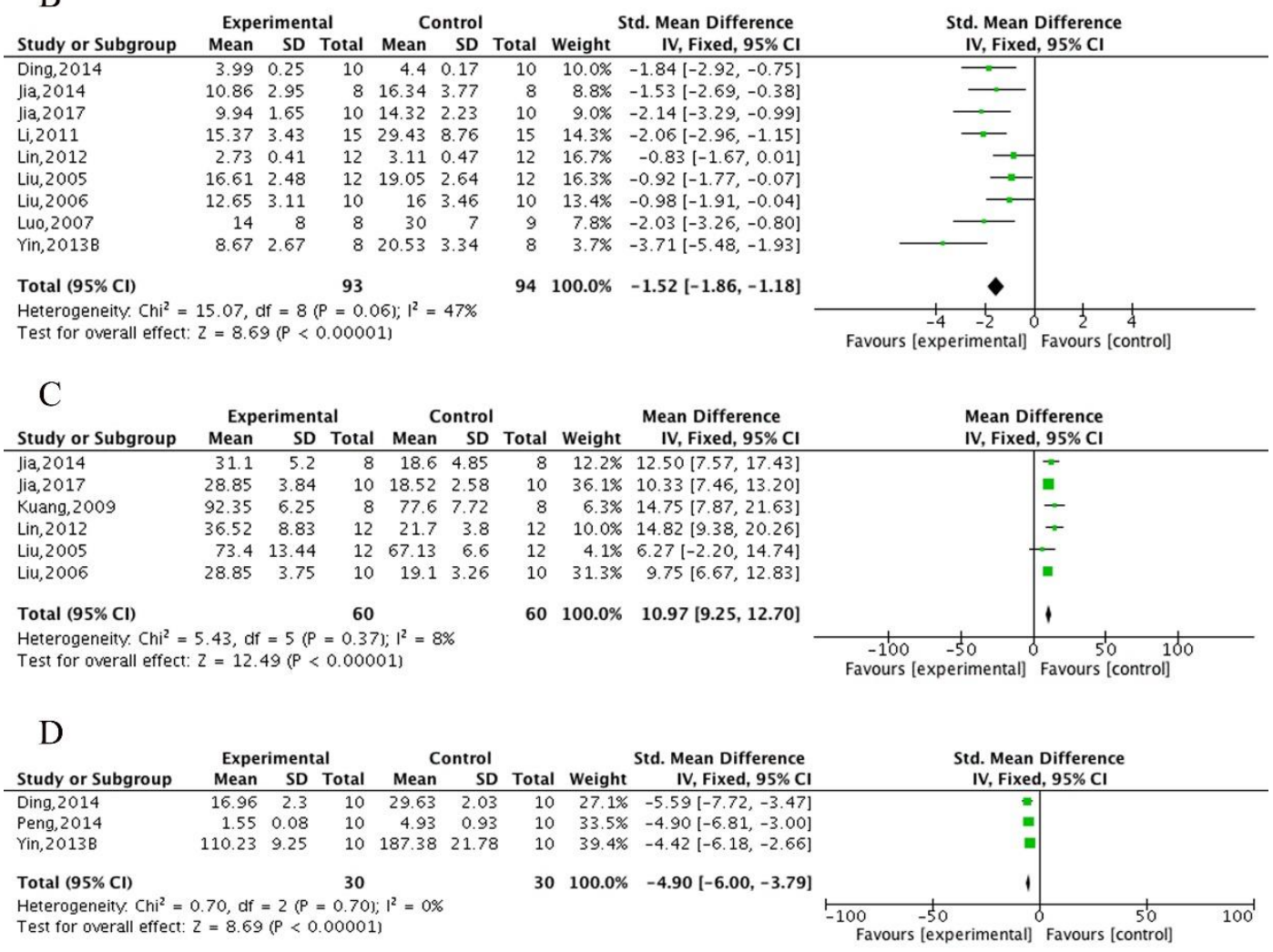

Figure 5. The forest plot of oxidative stress. Effects of ECC for increasing the activity of (A) SOD and (C) GSH-Px, decreasing (B) MDA and (D) NO compared with control group. 


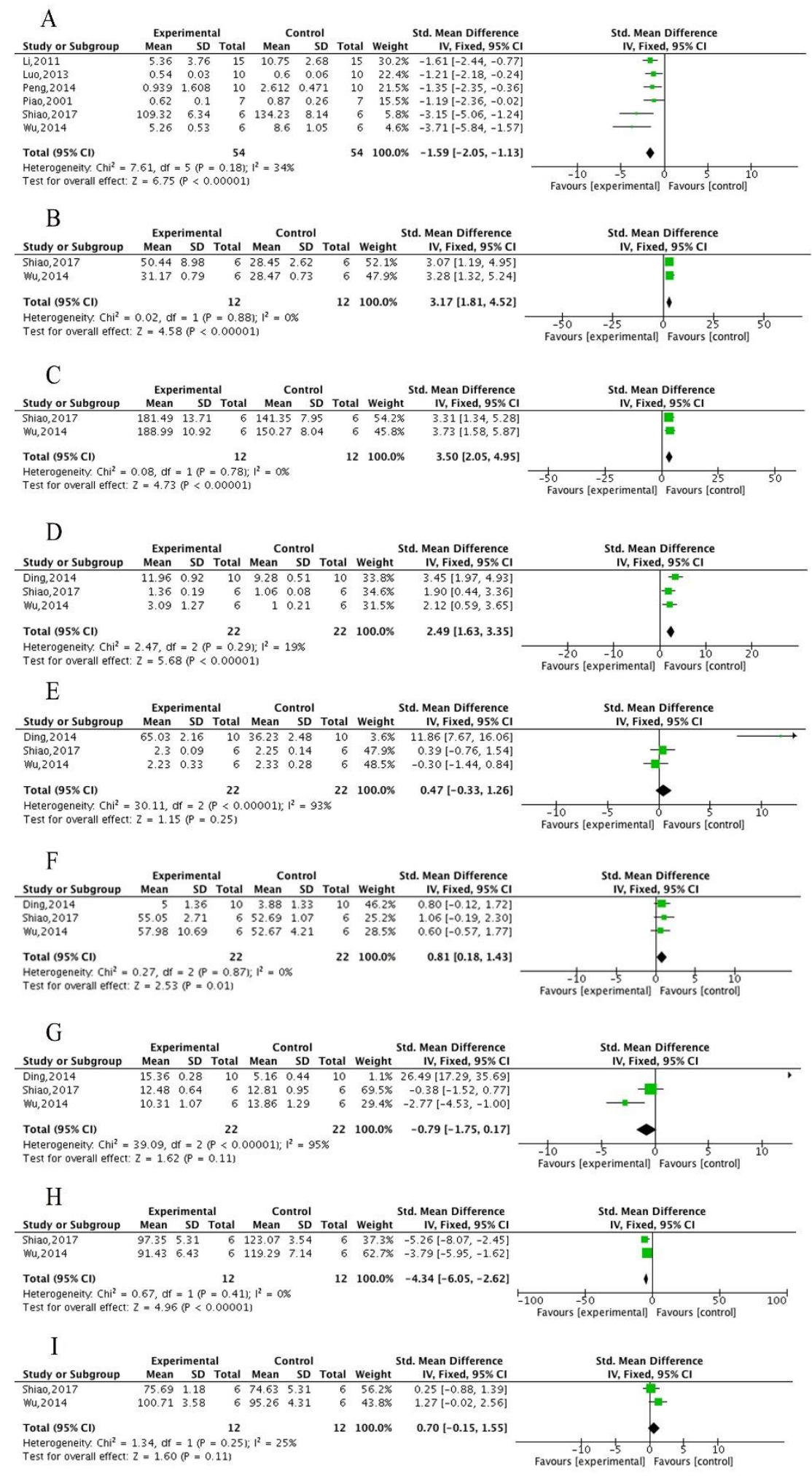

Figure 6. The forest plot of AChE and neurotransmitters. Effects of ECC for (A) decreasing the activity of AChE, increasing the level of Ach in hippocampus (B)/in cortex (C), increasing the level of DA in hippocampus (D)/in cortex (E), increasing the level of $\mathrm{NE}$ in hippocampus (F)/in cortex (G), and decreasing the activity of MAO-A in hippocampus (I)/in cortex (H) compared with control group. 
Table 2. Risk of bias of the included studies.

\begin{tabular}{|c|c|c|c|c|c|c|c|c|c|c|}
\hline Study & $\mathbf{A}$ & B & $\mathrm{C}$ & D & $\mathbf{E}$ & $\mathbf{F}$ & G & H & I & Total \\
\hline $\begin{array}{l}\text { Kuang, } \\
2009\end{array}$ & - & + & + & - & + & - & - & + & + & 5 \\
\hline Wu, 2014 & + & + & + & - & - & - & - & + & + & 5 \\
\hline Liu, 2005 & + & - & + & - & + & - & - & - & + & 4 \\
\hline Liu, 2006 & + & - & + & - & + & - & - & - & + & 4 \\
\hline Luo, 2007 & + & - & + & - & + & - & - & - & + & 4 \\
\hline Luo, 2013 & + & - & - & - & + & - & - & - & + & 3 \\
\hline $\begin{array}{l}\text { Yin, } 2013 \\
\text { (A) }\end{array}$ & + & - & + & - & + & + & - & - & + & 5 \\
\hline $\begin{array}{l}\text { Yin, } 2013 \\
\text { (B) }\end{array}$ & + & - & + & - & + & $?$ & - & - & + & 3 \\
\hline Li, 2011 & + & - & + & - & + & + & - & + & + & 6 \\
\hline Ding, 2014 & + & + & + & - & + & - & - & + & + & 6 \\
\hline Peng, 2015 & + & + & + & - & + & + & - & + & + & 7 \\
\hline Hu, 2016 & + & + & + & - & + & $?$ & - & + & + & 6 \\
\hline Jia, 2014 & + & + & + & - & + & + & - & + & + & 7 \\
\hline Jia, 2017 & + & + & + & - & + & + & - & + & + & 7 \\
\hline Gao, 2005 & + & - & + & - & + & + & - & - & + & 5 \\
\hline Wu, 2017 & + & - & + & - & + & + & - & + & + & 6 \\
\hline Yin, 2014 & + & - & + & - & + & + & - & - & + & 5 \\
\hline Shiao, 2017 & + & + & + & - & - & - & - & + & + & 5 \\
\hline Piao, 2001 & + & - & + & - & + & - & - & - & + & 4 \\
\hline Lin, 2012 & + & - & + & - & + & - & - & - & + & 4 \\
\hline
\end{tabular}

Note. Studies fulfilling the criteria of A: peer reviewed publication; B: control of temperature; C: random allocation to treatment or control; D: blinded induction of model or outcome; $\mathrm{E}$ : use of anesthetic without significant intrinsic neuroprotective activity; F: animal model (aged or female involved); G: sample size calculation; $\mathrm{H}$ : compliance with animal welfare regulations; I: statement of potential conflict of interests. $+=$ Yes, - = No, ? = unclear.

The step-down test, including the training test for learning score and retention test for memory score, was conducted in 10 studies [32, 34-37, 42, 46, 48, 50, 51]. Meta-analysis of 8 studies [32, 34-36, 37, 42, 48, 50] showed ECC were significant for decreasing the error times $(\mathrm{n}=167, \mathrm{MD}=-1.26,95 \% \mathrm{CI}[-1.60$ to -0.91$]$, $P<0.00001$; heterogeneity: $\chi^{2}=12.70, \mathrm{df}=7(P=0.08)$; $I^{2}=45 \%$; Fig. 3A), 3 studies $[32,36,42]$ for decreasing wrong reaction latency $(\mathrm{n}=71, \mathrm{MD}=0.56,95 \% \mathrm{CI}[0.08$ to 1.04], $P=0.02$; heterogeneity: $\chi^{2}=1.05$, df $=2(P=$ $0.59) ; I^{2}=0 \%$; Fig. 3B), 2studies [37, 48] for increasing right reaction latency $(P<0.05), 8$ studies $[32,34,35,42$, $46,48,50,51]$ for decreasing error times in the retention test $(\mathrm{n}=186, \mathrm{MD}=-1.35,95 \% \mathrm{CI}[-1.68$ to -1.01$]$, $P<0.00001$; heterogeneity: $\chi^{2}=12.33$, df $=7(P=0.09)$; $I^{2}=43 \%$; Fig. 3C), 5 studies [32, 42, 46, 50, 51] for decreasing wrong reaction latency in the retention test $(n$
$=125, \mathrm{MD}=1.05,95 \% \mathrm{CI}[0.67$ to 1.44$], P<0.00001$; heterogeneity: $\chi^{2}=6.60, \mathrm{df}=4(P=0.16) ; I^{2}=39 \%$; Fig. 3D) and 1 study [48] for increasing right reaction latency in the retention test $(P<0.05)$ compared with controls. Meta-analysis of 3 studies [48, 50, 51] showed there were no significant differences in reaction latency $(\mathrm{n}=68, \mathrm{MD}$ $=-3.02,95 \% \mathrm{CI}[-23.88$ to 17.84$], P=0.78$; heterogeneity: $\chi^{2}=1.43, \mathrm{df}=2(P=0.49) ; I^{2}=0 \%$; Supplementary Fig. 1B) and error time decrease $(\mathrm{n}=68$, $\mathrm{MD}=-0.19,95 \% \mathrm{CI} \quad[-0.71$ to 0.34$], P=0.49$; heterogeneity: $\chi^{2}=1.71, \mathrm{df}=2(P=0.43) ; I^{2}=0 \%$; Supplementary Fig. 1C) in the retention test between the ECC group and WCM controls.

The electrical Y-maze test was conducted in 4 studies [34, 36, 37, 50]. Meta-analysis of 2 studies [36, 37] showed ECC were significant for decreasing error reaction times $(\mathrm{n}=37, \mathrm{MD}=-2.48,95 \% \mathrm{CI}[-3.39$ to $-1.57], P<0.00001$; heterogeneity: $\chi^{2}=0.24, \mathrm{df}=1(P=$ $0.63) ; I^{2}=0 \%$; Fig. 4A), 2 studies $[34,50]$ for increasing right reaction times $(\mathrm{n}=44, \mathrm{MD}=0.84,95 \% \mathrm{CI}[0.22$ to 1.47], $P=0.008$; heterogeneity: $\chi^{2}=0.76$, df $=1(P=$ $0.38) ; I^{2}=0 \%$; Fig. $\left.4 \mathrm{~B}\right)$ and 1 study [34] for increasing right reaction times in the retention test $(P<0.05)$ compared with controls. The step-through test was performed in 3 studies [32, 33, 49]. Meta-analysis of 3 studies showed ECC were significant for decreasing latency in the training test $(\mathrm{n}=94, \mathrm{MD}=0.66,95 \% \mathrm{CI}$ [0.24 to 1.08], $P=0.002$; heterogeneity: $\chi^{2}=2.40, \mathrm{df}=2$ $(P=0.18) ; I^{2}=41 \%$; Fig. $\left.4 \mathrm{C}\right)$ and in the retention test $(\mathrm{n}$ $=54, \mathrm{MD}=1.26,95 \% \mathrm{CI}[0.66$ to 1.86$], P<0.0001$; heterogeneity: $\chi^{2}=1.81$, $\mathrm{df}=1(P=0.18) ; I^{2}=45 \%$; Fig. 4D) and 1 study [32] showed ECC significantly decreased the number of errors in both the training test and the retention test $(P<0.05)$ compared with controls. The open field test was conducted in 2 studies [33, 49] that both clearly showed ECC increased the frequency of visits and time spent in the hole compared with controls $(P<0.05)$, while 1 study [40] indicated that ECC markedly reduced the escape latency and the number of errors $(P<0.01)$ compared with controls. However, compared with WCM controls, ECC were statistically less effective in increasing the frequency $(\mathrm{n}=64, \mathrm{MD}=-3.6,95 \% \mathrm{CI}$ [-4.49 to -2.7$], P<0.001$; heterogeneity: $\chi^{2}=1.10, \mathrm{df}=$ $1(P=0.29) ; I^{2}=9 \%$; Supplementary Fig. 1D) and time spent in the hole $(\mathrm{n}=64, \mathrm{MD}=-3.44,95 \% \mathrm{CI}[-4.36$ to -2.53], $P<0.001$; heterogeneity: $\chi^{2}=1.24$, df $=1(P=$ $0.26) ; I^{2}=20 \%$; Supplementary Fig. $1 \mathrm{E}$ ).

\section{$\underline{\text { Neuroprotective mechanisms }}$}

Compared with controls, meta-analysis of 10 studies [32, 34-36, 39-41, 44, 45, 51] showed significant effects of ECC in increasing the activity of SOD $(n=207, \mathrm{MD}=$ $1.74,95 \%$ CI [1.40 to 2.08 ] $P<0.00001$; heterogeneity: $\chi^{2}$ 
$=17.21, \mathrm{df}=9(P=0.05) ; I^{2}=48 \%$; Fig. 5A $)$ and 9 studies [34-36, 39-41, 44, 45, 51] in decreasing MDA $(\mathrm{n}=187$, $\mathrm{MD}=-1.52,95 \% \mathrm{CI}[-1.86$ to -1.18$], P<0.00001$; heterogeneity: $\chi^{2}=15.07, \mathrm{df}=8(P=0.06) ; I^{2}=47 \%$; Fig. 5B). Compared with controls, meta-analysis of 6 studies $[32,34,35,44,45,51]$ showed significant effects of ECC in increasing GSH-Px $(\mathrm{n}=120, \mathrm{MD}=10.97,95 \% \mathrm{CI}[9.25$ to 12.70], $P<0.00001$; heterogeneity: $\chi^{2}=5.43, \mathrm{df}=5(P$ $=0.37) ; I^{2}=8 \%$; Fig. 5C) and 3 studies $[39,41,42]$ in decreasing $\mathrm{NO}(\mathrm{n}=60, \mathrm{MD}=-4.90,95 \%$ CI $[-6.00$ to -3.79], $P<0.00001$; heterogeneity: $\chi^{2}=0.70, \mathrm{df}=2(P=$ $0.70) ; I^{2}=0 \%$; Fig. 5D). Meta-analysis of 2 studies [33,
51] showed there were insignificant differences between ECC groups and WCM controls in increasing the activity of both SOD $(\mathrm{n}=44, \mathrm{MD}=-2.93,95 \% \mathrm{CI}[-7.05$ to 1.18$]$, $P=0.16$; heterogeneity: $\chi^{2}=0.62, \mathrm{df}=1(P=0.43) ; I^{2}=$ $0 \%$; Supplementary Fig. $2 \mathrm{~A})$ and MDA (n $=44, \mathrm{MD}=$ $-0.17,95 \% \mathrm{CI}[-0.84$ to 0.49$], P=0.61$; heterogeneity: $\chi^{2}$ $=14.57, \mathrm{df}=1(P=0.0001) ; I^{2}=93 \%$; Supplementary Fig. 2B). One study indicated there were insignificant differences between ECC and WCM groups in both increasing GSH-Px [51] and decreasing NO [41].

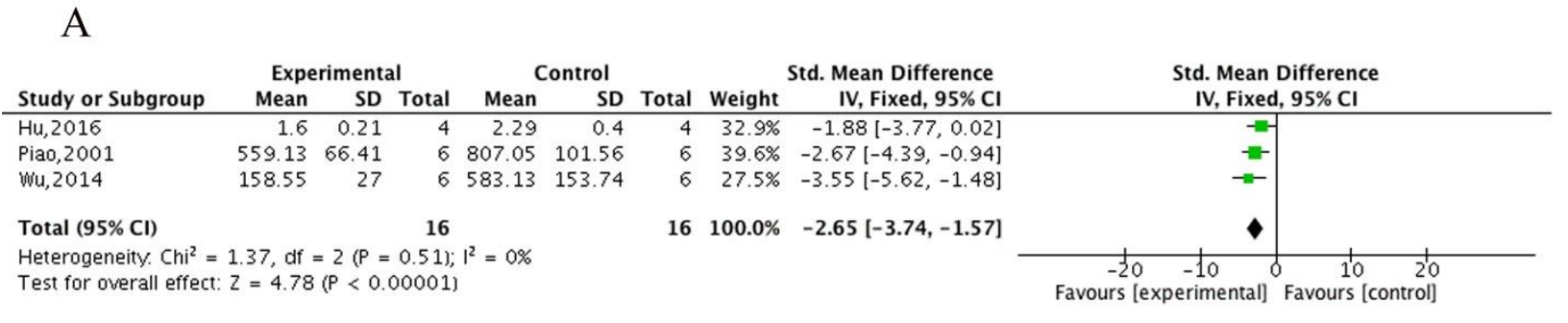

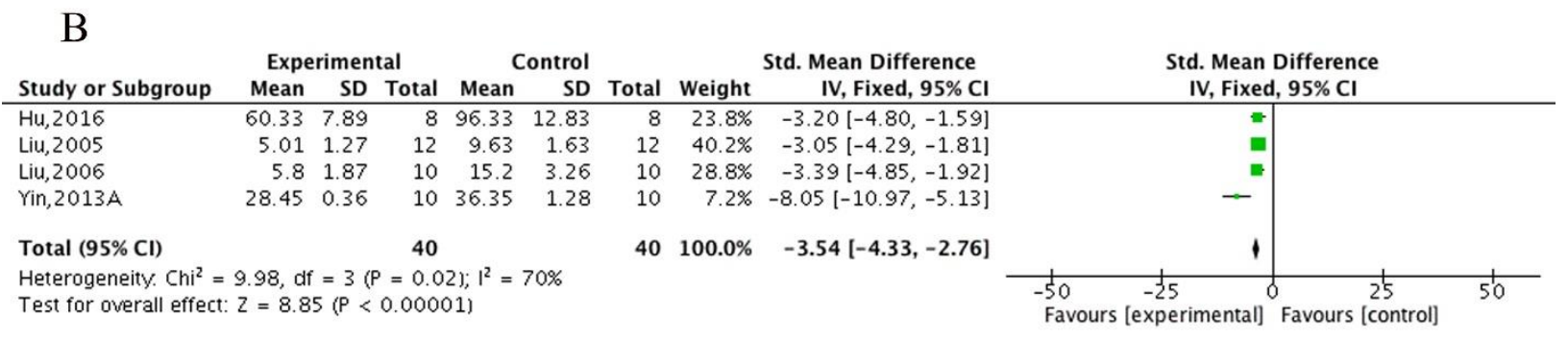

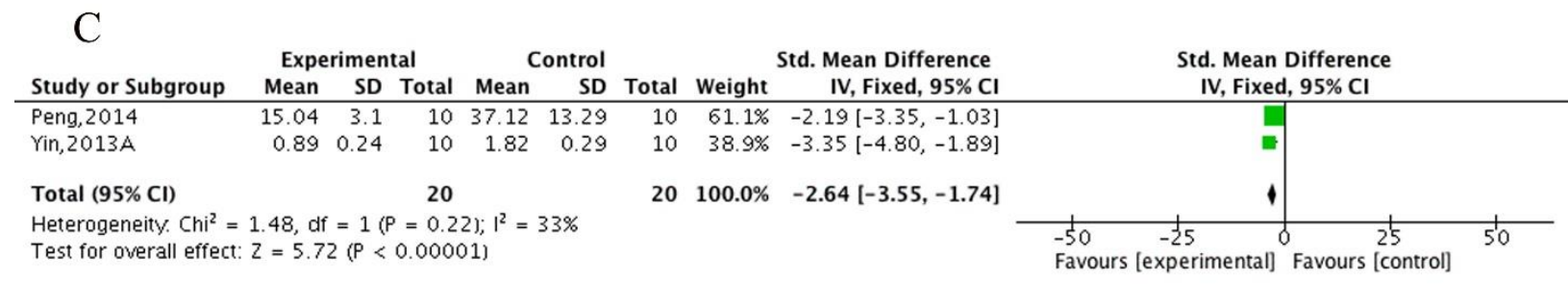

Figure 7. The forest plot of neuropathologic changes and Caspase-3. Effects of ECC for (A) decreasing A $\beta$ deposition, (B) decreasing apoptosis and $(\mathbf{C})$ decreasing Caspase-3compared with control group.

Compared with controls, meta-analysis of 6 studies $[33,37,40,42,49,50]$ showed ECC were significant for decreasing the activity of $\mathrm{AChE}(\mathrm{n}=108, \mathrm{MD}=-1.59$, 95\%CI [ -2.05 to -1.13 ],$P<0.00001$; heterogeneity: $\chi^{2}=$ $7.61, \mathrm{df}=5(P=0.18) ; I^{2}=34 \%$; Fig. 6A $), 2$ studies [33, 49] for increasing the level of Ach in both the hippocampus $(\mathrm{n}=24, \mathrm{MD}=3.17,95 \% \mathrm{CI}$ [1.81 to 4.52], $P<0.00001$; heterogeneity: $\chi^{2}=0.02, \mathrm{df}=1(P=0.88) ; I^{2}$
$=0 \%$; Fig. $6 \mathrm{~B})$ and the cortex $(\mathrm{n}=24, \mathrm{MD}=3.50,95 \% \mathrm{CI}$ [2.05 to 4.95], $P<0.00001$; heterogeneity: $\chi^{2}=0.08, \mathrm{df}=$ $1(P=0.78) ; I^{2}=0 \%$; Fig. 6C), 3 studies [33, 41, 49] for increasing the level of DA in the hippocampus $(n=44$, $\mathrm{MD}=2.49,95 \% \mathrm{CI}[1.63$ to 3.35$], \quad P<0.00001$; heterogeneity: $\chi^{2}=2.47, \mathrm{df}=2(P=0.29) ; I^{2}=19 \%$; Fig. 6D) but caused an insignificant difference in DA level in the cortex (n $=44, \mathrm{MD}=0.47,95 \% \mathrm{CI}[-0.33$ to 1.26$], P$ 
$=0.25$; heterogeneity: $\chi^{2}=30.11, \mathrm{df}=2, P<0.00001 ; I^{2}=$ 93\%; Fig. 6E), 3 studies [33, 41, 49] for increasing the level of NE in the hippocampus $(\mathrm{n}=44, \mathrm{MD}=0.81$, 95\% CI [0.18 to 1.43 ], $P=0.01$; heterogeneity: $\chi^{2}=0.27$, df $=2(P=0.87) ; I^{2}=0 \%$; Fig. $\left.6 \mathrm{~F}\right)$ but caused an insignificant difference in NE level in the cortex $(n=44$, $\mathrm{MD}=-0.79,95 \% \mathrm{CI}[-1.75$ to 0.17$], P=0.11$; heterogeneity: $\chi^{2}=39.09, \mathrm{df}=2(P<0.00001) ; I^{2}=95 \%$; Fig. 6G) and 2 studies $[33,49]$ in decreasing the activity of monoamine oxidase A (MAO-A) in the cortex $(n=24$, $\mathrm{MD}=-4.34,95 \% \mathrm{CI}[-6.05$ to -2.62$], P<0.00001$; heterogeneity: $\chi^{2}=0.67, \mathrm{df}=1(P=0.41) ; I^{2}=0 \%$; Fig. $6 \mathrm{H})$ but not in the hippocampus ( $=24, \mathrm{MD}=0.7,95 \% \mathrm{CI}$ [ -0.15 to 1.55$], P=0.11$; heterogeneity: $\chi^{2}=1.34, \mathrm{df}=1$ $(P=0.25) ; I^{2}=25 \%$; Fig. 61$)$. The change in the activity of monoamine oxidase B (MAO-B) was insignificant in both the cortex and the hippocampus between the ECC group and control group [33, 49]. Compared with WCM controls, meta-analysis of 2 studies [33, 50] showed ECC were insignificant in the activity of $\mathrm{AChE}(\mathrm{n}=26, \mathrm{MD}=$
$0.09,95 \% \mathrm{CI}[-0.07$ to 0.25$], P=0.26$; heterogeneity: $\chi^{2}$ $=1.54, \mathrm{df}=1(P=0.22) ; I^{2}=35 \%$; Supplementary Fig. $2 C)$. Two studies $[33,41]$ indicated that there was no significant difference between the ECC group and WCM controls in the level of DA $(\mathrm{n}=32, \mathrm{MD}=0.14,95 \% \mathrm{CI}$ $[-0.61$ to 0.9$], P=0.71$; heterogeneity: $\chi^{2}=6.84, \mathrm{df}=1$ $(P=0.009) ; I^{2}=85 \%$; Supplementary Fig. $\left.2 \mathrm{D}\right)$ and NE (n $=32, \mathrm{MD}=-0.77,95 \% \mathrm{CI}[-2.10$ to 0.56$], P=0.26$; heterogeneity: $\chi^{2}=34.22$, df $=1(P<0.0001) ; I^{2}=97 \%$; SupplementaryFig. 2E) in the hippocampus; however, significant differences were found in the level of DA $(\mathrm{n}=$ $32, \mathrm{MD}=-0.92,95 \% \mathrm{CI}[-1.36$ to -0.48$], P<0.0001$; heterogeneity: $\chi^{2}=0.66$, df $=1(P=0.42) ; I^{2}=0 \%$; Supplementary Fig. $2 \mathrm{~F})$ and $\mathrm{NE}(\mathrm{n}=32, \mathrm{MD}=-1.25$, 95\% CI [ -1.53 to -0.98$], P<0.0001$; heterogeneity: $\chi^{2}=$ 0.65 , df $=1(P=0.42) ; I^{2}=0 \%$; Supplementary Fig. $\left.2 \mathrm{G}\right)$ in the cortex. The levels of Ach, MAO-A and MAO-B were given in 1 study [33] and no significant differences were found between the ECC group and WCM controls.

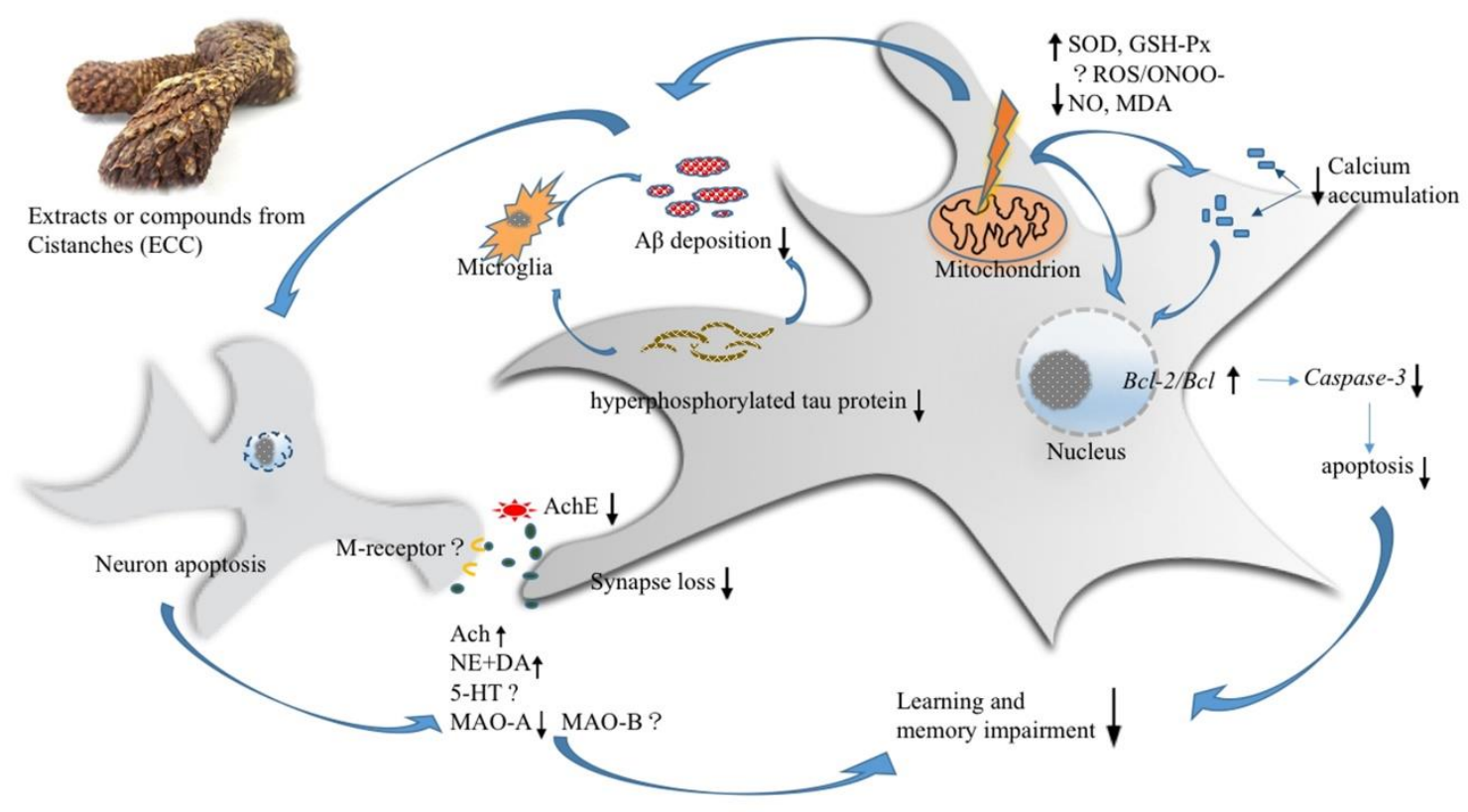

Figure 8. Summary of the possible neuroprotective mechanism of ECC for AD. ECC reduced the excessive ROS in mitochondrion, increased the activity of GSH-PX, SOD, and decreased the level NO and MDA. ECC decreased the level NO, down-regulated the over activation of microglia, exerting potential inhibitory effects on microglia-involved neuro-inflammation. ECC decreased $A \beta$ deposition and tau protein hyper-phosphorylation. ECC decreased the activity of AchE and maintained the normal level of Ach and NE in Cholinergic neuron and increased the level of DA in hippocampus. ECC activated the NMDA receptor and ameliorated the loss of synapses. The evidence of ECC in increasing the level of 5-HT is inadequate currently. ECC regulate the calcium deposition and maintain neuronal calcium homeostasis. ECC up-regulate the expressions of Bcl-2, decrease the ratio of $\mathrm{Bax} / \mathrm{Bcl}$, down-regulate the expressions of Caspase- 3 and reduce neurocyte apoptosis.

Three studies [33, 43, 49] showed ECC were marked in decreasing $\mathrm{A} \beta$ deposition $(\mathrm{n}=32, \mathrm{MD}=-2.65,95 \% \mathrm{CI}$ [-3.74 to -1.57$], P<0.00001$; heterogeneity: $\chi^{2}=1.37$, df
$=2(P=0.51) ; I^{2}=0 \% ;$ Fig. $\left.7 \mathrm{~A}\right), 4$ studies $[34,35,38$, $43]$ in decreasing apoptosis $(\mathrm{n}=80, \mathrm{MD}=-3.54,95 \% \mathrm{CI}$ [-4.33 to -2.76$], P<0.00001$; heterogeneity: $\chi^{2}=9.98$, df 
$=3(P=0.02) ; I^{2}=70 \%$; Fig. 7B $)$ and 2 studies $[38,42]$ in decreasing Caspase-3 $(\mathrm{n}=40, \mathrm{MD}=-2.64,95 \% \mathrm{CI}$ [ -3.55 to -1.74$], P<0.00001$; heterogeneity: $\chi^{2}=1.48$, $\mathrm{df}=1(P=0.22) ; I^{2}=33 \%$; Fig. $\left.7 \mathrm{C}\right)$. Two studies $[34,37]$ in decreasing of calcium deposition in the ECC group, with 1 study [37] indicating an increase in synapse number rather than a change in extrinsic features. Two studies [35, 38] obviously showed ECC increased the expression of Bcl-2 compared with controls. One study [33] provided data of the ECC and WCM control groups on $\mathrm{A} \beta$ deposition, but no intergroup differences were found. To summarize, we present a schematic overview of the neuroprotective mechanisms of ECC in AD (Fig. $8)$.

\section{DISCUSSION}

\section{Summary of evidence}

This is the first preclinical systematic review to assess the efficacy of ECC for experimental AD. Twenty studies with 1696 rodents were selected. The quality of the studies included ranged from 4 to 7 . The evidence available from the present study showed that ECC improved cognitive function in experimental AD mainly through mechanisms involving antioxidant stress and antiapoptosic effects, inhibiting $\mathrm{A} \beta$ deposition and tau protein hyperphosphorylation and promoting synapse protection.

\section{Limitations}

First, we only searched English and Chinese studies, which may lead to a certain degree of selective reporting and publication bias. It is well known that negative findings are less likely to be published. In the present analysis, some primary studies didn't provide original data, and some information was inaccessible. Thus, the dominance of positive studies might lead to the efficacy of ECC being overestimated. Second, the study quality was considered moderate, ranging from 4 to 7 out of 9 with a mean score of 5.05, indicating that the results should be explained with caution. Third, heterogeneity may exist due to the variety of $\mathrm{AD}$ model selection and preparation. Also, people with AD are always of old age, and a gender difference is observed in $\mathrm{AD}$ prevalence. In the present analysis, most $\mathrm{AD}$ models used healthy male rodents, which may lead to some challenges in clinical application.

\section{Implications for practice}

Preclinical animal research is the foundation of understanding of human diseases [52-54]; however, original preclinical research is often conducted with a small sample size, leading to less solid conclusions and poor repeatability [55]. The systematic review can integrate comprehensive preclinical evidence efficiently and guide potential clinical translation [56, 57]. The present study showed ECC could improve cognitive function and exert potential neuroprotective effects in experimental $\mathrm{AD}$ according to a large amount of experimental animal data, with 1696 rodents, indicating that ECC are candidates for AD treatment and can be used for further clinical trials. Besides, systematic review of animal researches is a more economical and ethical method to integrate preclinical evidence, helping to reduce unnecessary sacrifice of laboratory animals and preventing invalid or less informative researches [58, 59]. Systematic review of preclinical researches can identify defects in study design and implementation, contributing to improvement of the quality of follow-up preclinical researches $[60,61]$. In the present analysis, the quality of included studies ranged from 4 to 7 out of 9 points. The main flaws were a lack of sample size calculation, poor blinding in model induction and outcome assessment and an establishment of $\mathrm{AD}$ models based on no comorbidities. Reporting guidelines, such as ARRIVE, can provide guidance on the complete and transparent reporting of in vivo animal researches regularly and scientifically, helping to improve the quality of further researches [62-65]. Thus, we suggest that further animal researches of $\mathrm{AD}$ should follow up the reporting guidelines, increasing the value of clinical trials and further application.

Animal models are essential for understanding the induction and pathogenesis of a disease and developing therapeutic strategies that limit disease progression and eventually lead to effective treatments for the disease [66, 67]. An ideal AD model is essential for preclinical research and should include the following points: (1) correspondence to AD pathogenesis; (2) stable cognitive impairment; (3) low mortality; (4) simple to operate [68, 69]. In the present study, various kinds of AD models were used, including $A \beta$ cerebral ventricle infusion, $D-$ gal, scopolamine, sodium nitrite, $\mathrm{AlCl}_{3}$ or quinolinic acid intraperitoneal injection, and using SAMP8 mice and APP/PS1 transgenic mice. The former two kinds of AD models are more cost-effective and accessible, and are widely used in experimental AD research currently [70]; however, they can only partly simulate the pathological features and memory impairment symptoms of $\mathrm{AD}$. In addition, injection injury and ischemia or anoxia in multiple local organs is inevitable. Based on the aging comorbidity and pathology of AD, SAMP8 mice and APP/PSI double transgenic rats are better to mimic the characteristics of $\mathrm{AD}$ [71]; however, the weaknesses are a longer preparation time and a higher cost, which to a great 
extent limits their current use in practice. Further research on ideal AD model is urgently needed, which may also be of great importance in data analysis and preclinical evidence assessment.

The present study demonstrated ECC had neuroprotective effects in AD models according to the neurobehavioral, neurobiochemical and neuropathological observations. The mechanisms of ECC for AD are summarized as follows: (1) Antioxidant stress: ECC passed through the injured membrane, affecting the signal pathway of reactive oxygen species (ROS). ECC reduced the amount of excessive ROS in the mitochondrion, increased the activity of GSH-Px, SOD and sodium-potassium adenosine triphosphatase $\left(\mathrm{NA}^{+}-\right.$ $\mathrm{K}^{+}$ATPase) and decreased $\mathrm{NO}$ and MDA levels [39, 72, 73]. (2) Regulation of neuroinflammation: ECC decreased the level NO and down-regulated the over-activation of microglia, exerting potential inhibitory effects on microglia involving neuroinflammation $[74,75]$. In the included studies, the effects on neuroinflammation of $\mathrm{ECC}$ in $\mathrm{AD}$ are less pronounced, indicating modification in further research is needed. (3) Resisting $A \beta$ deposition and tau protein hyperphosphorylation: ECC decreased $\mathrm{A} \beta$ deposition and tau protein hyperphosphorylation [33, 43, 49], which may have an effect on oxidant stress and neuroinflammation; however, evidence on how ECC inhibit $\mathrm{A} \beta$ deposition and tau protein hyperphosphorylation is lacking, and further preclinical researches in vitro are essential. (4) Synapse protection: ECC decreased the activity of AchE, maintained normal Ach and NE levels in cholinergic neurons and increased the level of DA in the hippocampus. ECC activated the NMDA receptor and ameliorated the loss of synapses [48, 50], helping to regulate the proper function of synapses and guarantee essential intercellular contacts. However, evidence on the influence of ECC in increasing the level of 5-hydroxytryptamine (5-HT) in the brain is inadequate currently [41]. (5) Antiapoptosis: ECC can maintain the mitochondrial membrane potential and reduce the amount of excessive ROS, inhibiting the initiation of neural apoptosis $[32,76]$. ECC can up-regulate the expression of $\mathrm{Bcl}-2$, decrease the ratio of $\mathrm{Bax} / \mathrm{Bcl} 2$, down-regulate the expression levels of Caspase-3, P53, P65, SYN, PSD-95 and iNOS [45, 77] and reduce neuron apoptosis eventually. (6) Maintaining neuronal calcium homeostasis: An abnormal calcium steady state is the final common pathway of neuron destruction and is connected to oxidant stress, neuroinflammation, $A \beta$ deposition and tau protein hyperphosphorylation [78, 79]. ECC can protect the neuronal membrane, regulate the opening of calcium channels and maintain neuronal calcium homeostasis [34, 37, 80]. Further researches on calcium homeostasis and possible signal pathways are of great importance. In concluding, ECC act through complex, multicompound, multitarget and multipathway mechanisms in $\mathrm{AD}$ and might prove to be of great value in further clinical trials.

Animal experiments have contributed to our understanding of disease mechanisms, but the translation of preclinical experiments, which results in a prediction of the effectiveness of treatment strategies, to clinical trials is still challenging [81]. AD patients always have other medical problems such as aging, diabetes, hypertension and hyper lipidemia [82], and a gender difference is observed in AD prevalence [83]. The present study mainly included healthy male rats/mice, which may lead to selection bias to some extent. Registration of animal research prior to its execution in a generally accessible database similar to human (drug) research (www.clinicaltrials.com) may help to provide a more informed view before proceeding to clinical trials and may reduce publication bias $[69,81]$.

\section{Conclusions}

The present study showed ECC could improve cognitive function and exert potential neuroprotective effects in experimental $\mathrm{AD}$, largely through mechanisms involving antioxidant stress and antiapoptosic effects, inhibiting $A \beta$ deposition and tau protein hyperphosphorylation, and promoting synapse protection. Thus, ECC could be a candidate for further clinical trials of AD.

\section{Conflict of interest statement}

The authors declare no conflicts of interest.

\section{Acknowledgments}

This project was supported by the Young and MiddleAged University Discipline Leaders of Zhejiang Province, China (2013277); Zhejiang Provincial Program for the Cultivation of High-level Health Talents (2015). We would like to thank LetPub (www.letpub.com) for providing linguistic assistance during the preparation of this manuscript.

\section{Supplemenantry data}

The Supplemenantry data can be found online at: http://dx.doi.org/10.14336/AD.2018.0815-1

\section{References}

[1] Alzheimer A, Stelzmann RA, Schnitzlein HN, Murtagh FR (1995). An English translation of Alzheimer's 1907 paper, "Uber eine eigenartige Erkankung der Hirnrinde." Clin Anat, 8: 429-31. 
[2] McKhann GM (2011). Changing concepts of Alzheimer disease. JAMA, 305: 2458-9.

[3] Dubois B, Feldman HH, Jacova C, Hampel H, Molinuevo JL, Blennow K, et al. (2014). Advancing research diagnostic criteria for Alzheimer's disease: The IWG-2 criteria. Lancet Neurol, 13:614-29.

[4] Bassil N, Mollaei C (2012). Alzheimer's dementia: a brief review. J Med Liban, 60:192-9.

[5] Martin P, Maëlenn G, Matthew P, editors. Health Services \& Population Research. Alzheimer's Disease International (ADI), London; 2013.

[6] U.S. Alzheimer's Association (2017). 2017 Alzheimer's Disease Facts and Figures. Alzheimer's and Dementia, 13: 325-73.

[7] Ando K, Laborde Q, Lazar A, Godefroy D, Youssef I, Amar M, et al. (2014). Inside Alzheimer brain with CLARITY: senile plaques, neurofibrillary tangles and axons in 3-D. Acta Neuropathol, 128:457-9.

[8] Vinters HV (2015). Emerging Concepts in Alzheimer's Disease. Annual Review of Pathology: Mechanisms of Disease, 10: 291-319.

[9] Bloom GS (2014). Amyloid- $\beta$ and tau: the trigger and bullet in Alzheimer disease pathogenesis. JAMA Neurol, 1: 505-8.

[10] Visser PJ, Tijms B (2017). Brain Amyloid Pathology and Cognitive Function: Alzheimer Disease Without Dementia? JAMA, 317: 2285-7.

[11] Cummings JL, Zhong K (2014). Repackaging FDAapproved drugs for degenerative diseases: promises and challenges. Expert Rev Clin Pharmacol; 7:161-5.

[12] Qaseem A, Snow V, Cross JT Jr, Forciea MA, Hopkins R Jr, Shekelle P, et al.; American College of Physicians and American Academy of Family Physicians Panel on Dementia (2008). Current pharmacologic treatment of dementia: a clinical practice guideline from the American College of Physicians and the American Academy of Family Physicians. Ann Intern Med, 148: 370-8.

[13] Schmidt R, Hofer E, Bouwman FH, Buerger K, Cordonnier C, Fladby T, et al. (2015). EFNS-ENS/EAN Guideline on concomitant use of cholinesterase inhibitors and memantine in moderate to severe Alzheimer's disease. Eur J Neurol, 22: 889-98.

[14] Yang WT, Zheng XW, Chen S, Shan CS, Xu QQ, Zhu JZ, et al. (2017). Chinese herbal medicine for Alzheimer's disease: Clinical evidence and possible mechanism of neurogenesis. Biochem Pharmacol, 141: 143-55.

[15] Li JW, Zhou W, Li JS (2014). Original plant sources research of roucongrong in Chinese Pharmacopoeia. Chinese Archives of Traditional Chinese Medicine, 32: 1756-60.

[16] Wang T, Zhang X, Xie W (2012). Cistanche deserticola Y. C. Ma, "Desert ginseng": a review. Am J Chin Med, 40: 1123-41.

[17] Li Z, Lin H, Gu L, Gao J, Tzeng CM (2016). Herbal Cistanche (Rou Cong-Rong): One of the Best Pharmaceutical Gifts of Traditional Chinese Medicine. Front Pharmacol, 7: 41.
[18] Wang ZM, Liu XQ, Li C, Mao SJ (2017). Study on the history of drug and food dual use of Desert Cistanche. Chinese Pharmaceutical Journal, 52: 525-9.

[19] Pang F, Xu R, Xu CQ, Qi Yun, Shi Y, Ye ZG, et al. (2017). Research on medicinal and therapeutic history of Cistanche deserticola. Chinese Pharmaceutical Journal, 52: 377-83.

[20] Wang N, Ji S, Zhang H, Mei S, Qiao L, Jin X (2017). Herba Cistanches: Anti-aging. Aging Dis, 8: 740-59.

[21] Fu Z, Fan X, Wang X, Gao X (2017). Cistanches Herba: an overview of its chemistry, pharmacology, and pharmacokinetics property. J Ethnopharmacol,219:23347.

[22] Yang JH, Hu JP, Kasimu RN, Shu NS (2009). Study on the structure-activity relationship of the antioxidant activity of six phenylethanoid glycoside compounds in Cistanche. Chinese Medicinal Materials,32: 1067-9.

[23] Guo Q, Zhou Y, Wang CJ, Huang YM, Lee YT, Su MH, et al. (2013). An open-label, nonplacebo-controlled study on Cistanche tubulosa glycoside capsules (Memoregain (®)) for treating moderate Alzheimer's Disease. Am J Alzheimers Dis Other Demen, 28: 36370.

[24] Li N, Wang J, Ma J, Gu Z, Jiang C, Yu L, et al. (2015). Neuroprotective Effects of Cistanches Herba Therapy on Patients with Moderate Alzheimer's Disease. Evid Based Complement Alternat Med, 2015: 103985.

[25] Wang E, Huang Q, Huang MW, Bao ZX, Chen BJ, Wang Y, et al. (2011). Observation of curative effect of glycosides of Cistanche on Alzheimer's disease. Zhejiang Journal of Integrated Traditional Chinese and Western Medicine, 21: 699-701.

[26] Wang Q (2009). Clinical study on treatment of Alzheimer's disease with Cistanches glycosides. Strait Pharmaceutical Journal, 21: 103-4.

[27] Cuffy MC, Ratner LE, Siegler M, Woodle ES (2015). Equipoise: ethical, scientific, and clinical trial design considerations for compatible pair participation in kidney exchange programs. Am J Transplant, 15: 14849.

[28] Ritskes-Hoitinga M, Leenaars M, Avey M, Rovers M, Scholten R (2014). Systematic reviews of preclinical animal studies can make significant contributions to health care and more transparent translational medicine. Cochrane Database Syst Rev, 3: ED000078.

[29] Chen KY, Wang YN, Zhao YQ, Chen CH, Xu JK, Hu F, et al. (2015). Analyzing the Systematic Review/Metaanalysis of Animal Studies Published in Chinese Journals. Chinese Journal of Evidence-Based Medicine Magazine, 15: 414-8.

[30] Macleod MR, O'Collins T, Howells DW, Donnan GA (2004). Pooling of animal experimental data reveals influence of study design and publication bias. Stroke, 35:1203-8.

[31] Zheng XW, Yang WT, Chen S, Xu QQ, Shan CS, Zheng GQ, et al. (2017). Neuroprotection of Catalpol for Experimental Acute Focal Ischemic Stroke: Preclinical Evidence and Possible Mechanisms of Antioxidation, Anti-Inflammation, and Antiapoptosis. Oxid Med Cell Longev, 2017:5058609. 
[32] Kuang R (2009). The effect of glycosides of Cistanches on anti-oxidative stress in Alzheimer's disease model in vivo and vitro and its mechanism. Zhejiang University. www.wanfangdata.com.cn/details/detail.do?_type=degr ee\&id=Y1483057. Accessed 4.12, 2018.

[33] Wu CR, Lin HC, Su MH (2014). Reversal by aqueous extracts of Cistanche tubulosa from behavioral deficits in Alzheimer's disease-like rat model: relevance for amyloid deposition and central neurotransmitter function. BMC Complement Altern Med, 14: 202.

[34] Liu FX, Wang XW, Wang XF (2005). Effect of glycosides of Cistanches on learning and memory in Alzheimer's disease mice and its mechanism. Journal of Xin Jiang Medical University, 28: 1131-4.

[35] Liu FX, Wang XW, Luo L, Nabi XH, Wang XF (2006). The effect of glycosides of Cistanches on learning and memory in mice with Alzheimer's disease induced by beta amyloid peptide and its mechanism. China pharmacology, 5: 595-9.

[36] Luo L, Wang XW, Liu FX, Yang S, Wang T (2007). Effects of glycosides of Cistanches on behaviors and memory impairment mice induced by aluminum trichloride. Chinese drug and Clinical Journal, 1: 33-6.

[37] Luo L, Wu XC, Gao HJ, Lv SZ, Wang JH, Wang XW (2013). Effects of glycosides of Cistanches on neuroprotection in rats with Alzheimer's disease. Chinese pharmacies, 24: 2122-5.

[38] Yin G, Gong DK, Liu BH, Yao CJ (2013A). Effects of polysaccharides of Cistanches deserticola on learning and memory on Alzheimer's disease rats and the expression of Bcl-2 and caspase-3 in hippocampal neurons. Lishizhen medical medicine, 24: 1091-2.

[39] Yin G, Gong DK, Liu BH, Yao CJ (2013B). Effect of Polysacchrides of Cistanche Deserticola on learning and memory ability and oxygen stress of model rats with Alzheimer's disease, 30: 504-7.

[40] Li G (2011). Experimental study of the intelligence effect of Cistanches. Journal of Inner Mongolia Medical College, 33: 141-3.

[41] Ding H, Chen H, Jiang Y, Tu PF, Ma JY, Zhang WX (2014). The effects of echinacoside on improving the level of neurotransmitters in hippocampus and cortex of rats with Alzheimer's disease. Chinese pharmacology bulletin, 30: 1564-9.

[42] Peng XM, Gao L, Huo SX, Liu XM, Yan M (2015). The mechanism of memory enhancement of acteoside (verbascoside) in the senescent mouse model Induced by a Combination of D-gal and $\mathrm{AlCl} 3$. Phytother Res, 29: 1137-44.

[43] $\mathrm{Hu} \mathrm{H} \mathrm{(2016).} \mathrm{The} \mathrm{neuro-protective} \mathrm{effect} \mathrm{of} \mathrm{acteoside} \mathrm{in}$ mice of Alzheimer's disease. Journal of Liaoning University of Traditional Chinese Medicine, 18: 21-4.

[44] Jia JX, Song W, Yan XS, Yang ZJ, Fang X, Cai ZP, et al. (2014). The effect of glycosides of Cistanches on spacial learning and memory in SAMP8 mice and its mechanism. Journal of Baotou Medical College, 30: 6-8.

[45] Jia JX, Yan XS, Cai ZP, Song W, Huo DS, Zhang BF, et al. (2017). The effects of phenylethanoid glycosides, derived from Herba cistanche, on cognitive deficits and antioxidant activities in male SAMP8 mice. J Toxicol Environ Health A, 80:1180-6.

[46] Gao C, Wang CS, Wu GZ, Tu PF (2005). Glycosides of Cistanchis for mice with learning and memory impairment. New medicine and clinical pharmacology of traditional Chinese Medicine, 16: 162-4.

[47] Wu Y, Zhang H, Bu R, Ma H, Su M, Li G (2017). The protective effect of polysaccharide of Cistanches on acute senescence model induced by D-galactose. Chinese Pharmacological Bulletin, 33: 927-33.

[48] Yin RX, Li G, Yu TF, Ma H, Ma TY, Guo M (2014). Protective effects of polysaccharide of Cistanches deserticola on synaptic plasticity in learning and memory impaired mice induced by scopolamine. Chinese pharmacology bulletin, 30: 801-7.

[49] Shiao YJ, Su MH, Lin HC, Wu CR (2017). Echinacoside ameliorates the memory impairment and cholinergic deficit induced by amyloid beta peptides via the inhibition of amyloid deposition and toxicology. Food Funct, 8: 2283-94.

[50] Pu JH, Pu XP, Ma J, Li CL, Song ZH, Tu PF (2001). Effect of acetoside on improving the ability of learning and memory in mice. China Pharmacological Bulletin, 17: 625-7.

[51] Lin J, Gao L, Huo SX, Peng XM, Wu PP, Cai LM, et al. (2012). Effect of acteoside on learning and memory impairment induced by scopolamine in mice. Chinese Journal of traditional Chinese medicine, 37: 2956-9.

[52] Rappaport BA, Suresh S, Hertz S, Evers AS, Orser BA (2015). Anesthetic neurotoxicity--clinical implications of animal models. N Engl J Med, 372: 796-7.

[53] Careaga M, Murai T, Bauman MD (2017). Maternal Immune Activation and Autism Spectrum Disorder: From Rodents to Nonhuman and Human Primates. Biol Psychiatry, 81:391-401.

[54] Yardley MM, Ray LA (2017). Medications development for the treatment of alcohol use disorder: insights into the predictive value of animal and human laboratory models. Addict Biol, 22: 581-615.

[55] van der Worp HB, Macleod MR (2011). Preclinical studies of human disease: time to take methodological quality seriously. J Mol Cell Cardiol, 51: 449-50.

[56] Macleod MR, Ebrahim S, Roberts I (2005). Surveying the literature from animal experiments: systematic review and meta-analysis are important contributions. BMJ, 331:110.

[57] Shinohara M, Kanekiyo T, Yang L, Linthicum D, Shinohara M, Fu Y, et al. (2016). APOE2 eases cognitive decline during Aging: Clinical and preclinical evaluations. Ann Neurol, 79: 758-74.

[58] Felizardo AA, Marques DVB, Caldas IS, Gonçalves RV, Novaes RD (2018). Could age and aging change the host response to systemic parasitic infections? A systematic review of preclinical evidence. Exp Gerontol, 104:17-27.

[59] Barron CC, Lalu MM, Stewart DJ, Fergusson D, Yang H, Moher D, et al.; Canadian Perioperative Anesthesia Clinical Trials Group (2017). Assessment of safety and efficacy of mesenchymal stromal cell therapy in preclinical models of acute myocardial infarction: a systematic review protocol. Syst Rev226. 
[60] Macleod MR (2014). Preclinical research: Design animal studies better. Nature, 510: 35 .

[61] Xu JK, Zhao W, Liao XL, Wang H, Yan Q, Wu KN, et al. (2017). Evidence-based construction of animal experiment system evaluation process. Chinese Journal of Evidence-Based Medicine, 17:1357-64.

[62] Kilkenny C, Browne W, Cuthill IC, Emerson M, Altman DG; National Centre for the Replacement, Refinement and Reduction of Amimals in Research (2011). Animal research: reporting in vivo experiments--the ARRIVE guidelines. J Cereb Blood Flow Metab, 31: 991-3.

[63] Glasziou P, Altman DG, Bossuyt P, Boutron I, Clarke M, Julious S, et al. (2014). Reducing waste from incomplete or unusable reports of biomedical research. Lancet, 383: 267-76.

[64] Karp NA, Meehan TF, Morgan H, Mason JC, Blake A, Kurbatova N, et al. (2015). Applying the ARRIVE Guidelines to an In Vivo Database. PLoS Biol, 13: e1002151.

[65] Smith AJ, Clutton RE, Lilley E, Hansen KEA, Brattelid $T$ (2018). Improving animal research: PREPARE before you ARRIVE. BMJ, 360: k760.

[66] Duyckaerts C, Potier MC, Delatour B (2008). Alzheimer disease models and human neuropathology: similarities and differences. Acta Neuropathol, 115: 5-38.

[67] Robinson AP, Harp CT, Noronha A, Miller SD (2014). The experimental autoimmune encephalomyelitis (EAE) model of MS: utility for understanding disease pathophysiology and treatment. Handb Clin Neurol, 122: 173-89.

[68] Laurijssens B, Aujard F, Rahman A (2013). Animal models of Alzheimer's disease and drug development. Drug Discov Today Technol, 10: e319-27.

[69] Zheng Q, Bao XY, Zhu PC, Tong Q, Zheng GQ, Wang $Y$ (2017). Ginsenoside Rb1 for Myocardial Ischemia/Reperfusion Injury: Preclinical Evidence and Possible Mechanisms. Oxid Med Cell Longev, 2017: 6313625.

[70] Huang FJ, Cheng DJ, Wu ZZ, Qin J (2017). Research progress on pathological mouse model of Alzheimer's disease. Medical Recapitulate, 23: 4851-7.

[71] Porquet D, Andrés-Benito P, Griñán-Ferré C, Camins A, Ferrer I, Canudas AM, et al. (2015). Amyloid and tau pathology of familial Alzheimer's disease APP/PS1 mouse model in a senescence phenotype background (SAMP8). Age (Dordr), 37: 9747.

[72] Dumont M, Beal MF (2011). Neuroprotective strategies involving ROS in Alzheimer disease. Free Radic Biol Med, 51: 1014-26.
[73] Chen W, Lin HR, Wei CM, Luo XH, Sun ML, Yang ZZ, et al. (2018). Echinacoside, a phenylethanoid glycoside from Cistanche deserticola, extends lifespan of Caenorhabditis elegans and protects from $A \beta$-induced toxicity. Biogerontology, 19: 47-65.

[74] Gutierrez A, Vitorica J (2018). Toward a New Concept of Alzheimer's Disease Models: A Perspective from Neuroinflammation. J Alzheimers Dis, 64(s1): S329-38.

[75] Nan ZD, Zeng KW, Shi SP, Zhao MB, Jiang Y, Tu PF (2013). Phenylethanoid glycosides with antiinflammatory activities from the stems of Cistanche deserticola cultured in Tarim desert. Fitoterapia, 89: 16774.

[76] Meng SX, Huo QP (2016). Research progress on the effect of active ingredients of Cistanche on nervous system. Chinese Journal of Information on Traditional Chinese Medicine, 23: 123-6.

[77] Xu Q, Fan W, Ye SF, Cong YB, Qin W, Chen SY, et al. (2016). Cistanche tubulosa Protects Dopaminergic Neurons through Regulation of Apoptosis and Glial CellDerived Neurotrophic Factor: in vivo and in vitro. Front Aging Neurosci, 8: 295.

[78] Popugaeva E, Pchitskaya E, Bezprozvanny I (2017). Dysregulation of neuronal calcium homeostasis in Alzheimer's disease - A therapeutic opportunity? Biochem Biophys Res Commun, 483: 998-1004.

[79] Alzheimer's Association Calcium Hypothesis Workgroup (2017). Calcium Hypothesis of Alzheimer's disease and brain aging: A framework for integrating new evidence into a comprehensive theory of pathogenesis. Alzheimers Dement, 13: 178-82.

[80] Yao XM, Li XX, Zhou J, Wang Q, Liu GZ, Zhou Y (2018). Experimental research progress of calcium ion homeostasis intervention for Alzheimer's disease. Chinese Archives of Traditional Chinese Medicine, 36: 49-52.

[81] Hackam DG, Redelmeier DA (2006). Translation of research evidence from animals to humans. JAMA, 296: 1731-2.

[82] Fan YC, Hsu JL, Tung HY, Chou CC, Bai CH (2017). Increased dementia risk predominantly in diabetes mellitus rather than in hypertension or hyperlipidemia: a population-based cohort study. Alzheimers Res Ther, 9:7.

[83] Payami H, Zareparsi S, Montee KR, Sexton GJ, Kaye JA, Bird TD, et al. (1996). Gender difference in apolipoprotein e-associated risk for familial Alzheimer disease: a possible clue to the higher incidence of Alzheimer disease in women. Am J Hum Genet, 58: 803. 